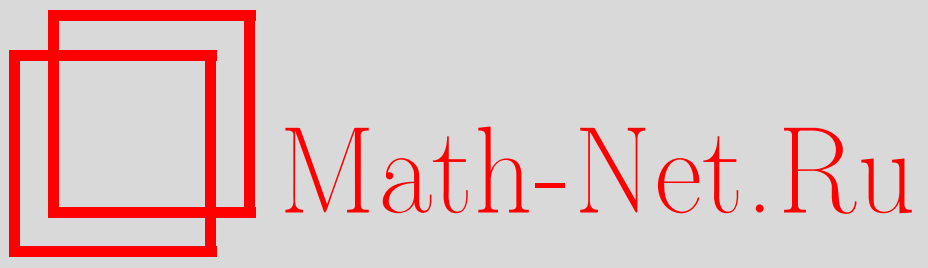

Б. Берджан, С. М. Пергаменщиков, Последовательные $\delta$ оптимальные потребление и инвестирование для финансовых рынков со стохастической волатильностью при неизвестных параметрах, Теория вероятн. и ее примен., 2015, том 60, выпуск 4, 628-659

DOI: https://doi.org/10.4213/tvp5029

Использование Общероссийского математического портала Math-Net.Ru подразумевает, что вы прочитали и согласны с пользовательским соглашением http://www . mathnet.ru/rus/agreement

Параметры загрузки:

IP: 54.147 .182 .235

26 апреля 2023 г., 11:31:45

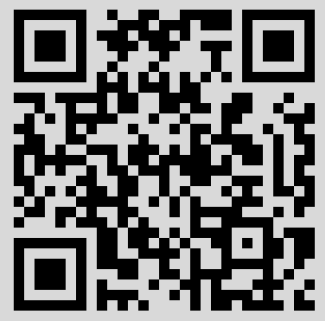




\title{
ПОСЛЕДОВАТЕЛЬНЫЕ $\delta$-ОПТИМАЛЬНЫЕ ПОТРЕБЛЕНИЕ И ИНВЕСТИРОВАНИЕ ДЛЯ ФИНАНСОВЫХ РЫНКОВ СО СТОХАСТИЧЕСКОЙ ВОЛАТИЛЬНОСТЬЮ ПРИ НЕИЗВЕСТНЫХ ПАРАMETPAX ${ }^{1)}$
}

\begin{abstract}
Рассматривается задача оптимального потребления и инвестирования для финансового рынка Блэка-Шоулса со стохастической волатильностью и с неизвестным коэффициентом роста акций. Параметр волатильности управляется внешним экономическим фактором, который моделируется процессом Орнштейна-Уленбека с неизвестным параметром сноса. В первой части статьи мы методом динамического программирования находим оптимальную финансовую стратегию, которая зависит от неизвестных параметров рынка. Чтобы оценить коэффициент сноса, мы наблюдаем процесс, моделирующий внешний экономический фактор $Y$, на интервале $\left[0, T_{0}\right]$ для заданного $T_{0}>0$ и пользуемся методом последовательного оценивания. Во второй части статьи мы показываем, что целевая функция для такой стратегии находится в $\delta$-окрестности ее оптимального значения, т.е. стратегия, построенная с помощью последовательного оценивания, является $\delta$-оптимальной.
\end{abstract}

Ключевые слова и фразы: последовательный анализ, усеченная последовательная оценка, модель Блэка-Шоулса, стохастическая волатильность, оптимальные потребление и инвестирование, уравнение Гамильтона-Якоби-Беллмана.

1. Введение. В данной работе изучается задача оптимального потребления и инвестирования для финансового рынка Блэка-Шоулса со случайной волатильностью (см., например, [7]). Мы рассматриваем

*Département de mathématiques, Université du Québec à Montréal, Montréal, Québec, Canada; email: berdjane_b@yahoo.fr

** Laboratoire de mathématiques Raphael Salem, Université de Rouen, Saint-Étienne du Rouvray, France; Международная лаборатория количественных финансов, Национальный исследовательский университет «Высшая школа экономики», Москва, Россия; e-mail: serge.pergamenchtchikov@univ-rouen.fr

1) Работа выполнена при поддержке Международной лаборатории количественных финансов (грант Правительства РФ № 14.А12.31.0007) и Российского научного фонда (проект № 14-49-00079, Московский энергетический институт). Второй автор поддержан также Международной лабораторией статистики случайных процессов и количественного финансового анализа Национального исследовательского Томского государственного университета. 
одну и ту же степенную функцию полезности для потребления и терминального капитала. Предполагается, что параметр волатильности зависит от некоторого экономического фактора, моделируемого процессом Орнштейна-Уленбека. Классический подход к этой проблеме восходит к работе Р. Мертона [22].

С использованием результатов теории стохастического управления явные решения были получены для финансовых рынков с неслучайными коэффициентами (см., например, [13], [15], [29], [26]). Далее задачи потребления и инвестирования обобщались во многих направлениях [27]. Одним из важных обобщений таких задач являются финансовые модели со стохастической волатильностью. Эмпирические исследования фондовых рынков показывают, что волатильность имеет случайный характер (см. [28] и [10]). Чисто инвестиционные проблемы для таких моделей рассматривались в работах [31] и [25], где с помощью динамического программирования было показано, что нелинейное уравнение Гамильтона-Якоби-Беллмана (НJB) может быть преобразовано в квазилинейное уравнение в частных производных. Аналогичный подход был использован в [16] для задачи оптимального потребления и инвестирования с учетом разорения в случае финансовых рынков с неслучайными коэффициентами. В [5] с использованием гирсановской замены меры изучалась задача оптимального потребления (без инвестирования) для финансовых рынков со стохастической волатильностью. В работах [2] и [9] использовались дуальные методы. Как правило, теорема существования и единственности для уравнения НЈВ доказывается с помощью классических методов линейных уравнений в частных производных (см., например, [6, гл. VI.6 и приложение Е]). В настоящей статье мы используем подход, предложенный в [4] и развитый в [1]. Разница заключается в том, что в [4] экономический фактор моделируется чисто скачкообразным процессом. Уравнение НЈВ в этом случае является интегро-дифференциальным уравнением первого порядка. В нашем же случае мы имеем существенно нелинейное уравнение в частных производных второго порядка. В работе [1] эта задача рассматривалась с известными параметрами модели рынка и в предположении, что внешний экономический фактор моделируется диффузионным процессом с ограниченными коэффициентами. Последнее условие исключает из рассмотрения процесс Орнштейна-Уленбека, используемый во многих практически важных моделях со случайной волатильностью. В данной работе, так же как в [4] и [1], уравнение НJВ изучается с помощью формулы Фейнмана-Каца. Мы вводим специальное метрическое пространство, в котором оператор Фейнмана-Каца является сжимающим. Принимая это во внимание, мы доказываем теорему о неподвижной точке для этого оператора и устанавливаем, что неподвижная точка является решением уравнения НЈВ в нашем случае. 
Во второй части статьи мы рассматриваем ситуацию, когда и коэффициент роста в уравнении для акций, и коэффициент сноса в уравнении для экономического фактора являются неизвестными. Чтобы оценить параметр сноса в процессе Орнштейна-Уленбека, мы используем методы последовательного анализа, предложенные в [23] и [19]. Параметр сноса будет оцениваться на основе наблюдений процесса $Y$ на интервале $\left[0, T_{0}\right]$. Следует отметить, что обычная оценка максимального правдоподобия в данном случае является нелинейной функцией от наблюдений и поэтому невозможно непосредственно оценить неасимптотическую верхнюю границу для ее точности. Чтобы преодолеть эту трудность, мы используем усеченную последовательную процедуру, предложенную в [14], которая позволяет получить неасимптотическую верхнюю границу для среднеквадратической точности оценивания. После этого мы пользуемся этой оценкой в оптимальной стратегии на интервале $\left[T_{0}, T\right]$. Для полученной стратегии мы устанавливаем, что математическое ожидание модуля отклонения целевой функции от ее максимального значения не превосходит некоторого известного фиксированного числа $\delta>0$, т.е. стратегия, вычисленная посредством последовательной процедуры, является $\delta$-оптимальной.

Статья организована следующим образом: в разделах 2 и 3 мы вводим модель рынка, ставим оптимизационную задачу и определяем соответствующее уравнение НЈВ. В разделе 4 вводятся необходимые определения. Решение задачи оптимального потребления и инвестирования дается в разделах 5-7. В разделе 8 мы рассматриваем задачу с неизвестным параметром сноса $\alpha$ в стохастическом уравнении для экономического фактора $Y$ и используем усеченную последовательную процедуру $\widehat{\alpha}$. Мы получаем явную верхнюю границу для отклонения $\mathbf{E}|\widehat{\alpha}-\alpha|$ при любом фиксированном $T_{0}>0$. Более того, используя эту усеченную оценку в оптимальной стратегии потребления и инвестирования на интервале $\left[T_{0}, T\right]$, мы показываем, что эта стратегия является $\delta$-оптимальной. Аналогичные результаты приведены в п. 8.3 для случая, когда кроме оценки $\widehat{\alpha}$ мы рассматриваем оценку $\widehat{\mu}$ неизвестного коэффициента роста в уравнении для акций. Численный пример приведен в разделе 9 . Все вспомогательные результаты, необходимые для изучения свойств непрерывности по параметрам решения уравнения Беллмана (см. предложения 10.1 и 10.2), вынесены в приложение (раздел 10).

2. Модель рынка. Пусть $\left(\Omega, \mathscr{F}_{T},\left(\mathscr{F}_{t}\right)_{0 \leqslant t \leqslant T}, \mathbf{P}\right)$ - стандартное фильтрованное вероятностное пространство с двумя заданными на нем независимыми винеровскими процессами $\left(W_{t}\right)_{0 \leqslant t \leqslant T}$ и $\left(\mathbf{U}_{t}\right)_{0 \leqslant t \leqslant T}$, принимающими значения в $\mathbf{R}$. Наш финансовый рынок состоит из одного нерискового актива $S_{0}=\left(S_{0}(t)\right)_{0 \leqslant t \leqslant T}$ и одной акиии $S=(S(t))_{0 \leqslant t \leqslant T}$, 
которые определяются следующими уравнениями:

$$
\begin{aligned}
& \mathrm{d} S_{0}(t)=r S_{0}(t) \mathrm{d} t \\
& \mathrm{~d} S(t)=S(t) \mu \mathrm{d} t+S(t) \sigma\left(Y_{t}\right) \mathrm{d} W_{t},
\end{aligned}
$$

где $S_{0}(0)=1$ и $S(0)=s>0$. В данной модели $r \in \mathbf{R}_{+}$- это прочентная ставка безрискового актива, $\mu$ - коэффициент роста и $\sigma(y)$ волатильность. Волатильность $\sigma$ является ограниченной функцией из $\mathbf{R}$ в $\mathbf{R}_{+}$и удовлетворяет соотношению

$$
\inf _{y \in \mathbf{R}} \sigma(y)=\sigma_{1}>0 .
$$

Мы предполагаем также, что $\sigma(\cdot)$ дифференцируема и имеет ограниченную производную. Более того, мы считаем, что стохастический фактор $Y$ со значениями в $\mathbf{R}$ задается процессом Орнштейна-Уленбека, определяемым линейным стохастическим уравнением

$$
\mathrm{d} Y_{t}=\alpha Y_{t} \mathrm{~d} t+\beta \mathrm{d} \mathbf{U}_{t},
$$

где начальное значение $Y_{0}$ - неслучайная константа, а $\alpha<0, \beta>0-$ некоторые фиксированные параметры. Пусть $\left(Y_{s}^{t, y}\right)_{s \geqslant t}$ обозначает процесс $Y$, подчиняющийся условию $Y_{t}=y$, т.е.

$$
Y_{s}^{t, y}=y e^{\alpha(s-t)}+\int_{t}^{s} \beta e^{\alpha(s-v)} \mathrm{d} \mathbf{U}_{v}
$$

В данной работе рассматривается оптимизационная задача на временном интервале $\left[T_{0}, T\right]$, где $0 \leqslant T_{0}<T$ - фиксированные временные границы. Пусть $X_{t}$ - капитал инвестора в момент времени $t \in\left[T_{0}, T\right]$. Обозначим через $\varphi_{t} \in \mathbf{R}$ долю капитала, вкладываемую в акции $(S)$, при этом $1-\varphi_{t}$ будет долей капитала, вкладываемой в нерисковый актив $\left(S_{0}\right)$. Стратегия инвестора в момент времени $t \in\left[T_{0}, T\right]$ состоит, во-первых, в выборе доли $\varphi_{t}$ и, во-вторых, в выборе интенсивности потребления $\varsigma_{t}\left(\varsigma_{t} \geqslant 0\right)$. Тогда в соответствии с моделью (2.1) эволюция капитала задается уравнением

$\mathrm{d} X_{t}=\mu \varphi_{t} X_{t} \mathrm{~d} t+\sigma\left(Y_{t}\right) \varphi_{t} X_{t} \mathrm{~d} W_{t}+r\left(1-\varphi_{t}\right) X_{t} \mathrm{~d} t-\varsigma_{t} \mathrm{~d} t, \quad T_{0} \leqslant t \leqslant T$,

где $X_{T_{0}}=x>0$ - начальный капитал. Заметим, что для модели (2.1) параметр риска - это функция из $\mathbf{R}$ в $\mathbf{R}$, определяемая формулой

$$
\theta(y)=\frac{\mu-r}{\sigma(y)}
$$

Если в качестве стратегии вместо пары $\left(\varphi_{t}, \varsigma_{t}\right)$ рассмотреть $\left(\pi_{t}, c_{t}\right)$, где $\pi_{t}=\sigma\left(Y_{t}\right) \varphi_{t}$ и $c_{t}=\varsigma_{t} X_{t}$, то процесс капитала будет удовлетворять следующему стохастическому дифференциальному уравнению:

$$
\mathrm{d} X_{t}=X_{t}\left(r+\pi_{t} \theta\left(Y_{t}\right)-c_{t}\right) \mathrm{d} t+X_{t} \pi_{t} \mathrm{~d} W_{t}, \quad X_{T_{0}}=x .
$$


Опишем множество допустимых стратегий. Переменная управления (финансовая стратегия) $\vartheta=\left(\vartheta_{t}\right)_{T_{0} \leqslant t \leqslant T}=\left(\left(\pi_{t}, c_{t}\right)\right)_{T_{0} \leqslant t \leqslant T}$ называется допустимой, если она $\left(\mathscr{F}_{t}\right)_{T_{0} \leqslant t \leqslant T}$-прогрессивно измерима, принимает значения в $\mathbf{R} \times[0, \infty)$ и для нее уравнение $(2.5)$ имеет единственное сильное почти наверное положительное решение $\left(X_{t}^{\vartheta}\right)_{T_{0} \leqslant t \leqslant T}$. Мы обозначим множество всех допустимьих стратегий через $\mathscr{V}$.

В этой статье мы рассматриваем степенные функции полезности $x^{\gamma}, 0<\gamma<1$, как для потребления, так и для терминального капитала. Цель заключается в максимизации усредненной интегральной полезности потребления на интервале $\left[T_{0}, T\right]$ при фиксированном $T_{0}$ и усредненной полезности для терминального капитала в последний момент $T$. Тогда для любых $x, y \in \mathbf{R}$ и $\vartheta \in \mathscr{V}$ целевая функция определяется формулой

$$
J\left(T_{0}, x, y, \vartheta\right):=\mathbf{E}_{T_{0}, x, y}\left(\int_{T_{0}}^{T} c_{t}^{\gamma}\left(X_{t}^{\vartheta}\right)^{\gamma} \mathrm{d} t+\left(X_{T}^{\vartheta}\right)^{\gamma}\right),
$$

где $\mathbf{E}_{T_{0}, x, y}$ - условное математическое ожидание $\mathbf{E}\left(\cdot \mid X_{T_{0}}=x, Y_{T_{0}}=y\right)$. Наша задача - максимизировать эту функцию, т.е. найти

$$
J\left(T_{0}, x, y, \vartheta^{*}\right)=\sup _{\vartheta \in \mathscr{V}} J\left(T_{0}, x, y, \vartheta\right) .
$$

В дальнейшем вместо $J\left(T_{0}, x, y, \vartheta^{*}\right)$ мы будем писать $J^{*}\left(T_{0}, x, y\right)$ или просто $J_{T_{0}}^{*}$.

В случае, когда параметры $\alpha$ и $\mu$ неизвестны, мы предполагаем, что $\alpha_{2} \leqslant \alpha \leqslant \alpha_{1}$ и $|\mu| \leqslant \mu_{*}$, где $\alpha_{2}<\alpha_{1}<0$ и $\mu_{*}>0$ - известные фиксированные константы. Наша задача состоит в том, чтобы построить стратегии, приближающиеся в определенном смысле к оптимальным, а именно, мы будем искать $\delta$-оптимальные стратегии в смысле следующего определения.

О п р е д е л е н и е 2.1. Стратегию $\widetilde{\vartheta} \in \mathscr{V}$ будем называть $\delta$ оптимальной, если

$$
\sup _{\alpha_{2} \leqslant \alpha \leqslant \alpha_{1}} \sup _{|\mu| \leqslant \mu_{*}} \mathbf{E}\left(J\left(T_{0}, x, Y_{T_{0}}, \widetilde{\vartheta}\right)-J^{*}\left(T_{0}, x, Y_{T_{0}}\right)\right) \leqslant \delta .
$$

3 а м е ч а н и е 2.1. Отметим, что если параметры $\alpha$ и $\mu$ известны, то мы рассматриваем задачу $(2.6)$ с $T_{0}=0$. Следует также отметить, что при известных параметрах эта задача решается в [1], но в качестве экономического фактора $Y$ там рассматривается общий процесс диффузии с ограниченными коэффициентами. В данной работе мы предполагаем, что $Y$ является процессом Орнштейна-Уленбека, т.е. снос неограничен и мы, к сожалению, не можем пользоваться результатами работы [1]. 
3. Уравнение Гамильтона-Якоби-Беллмана (НJВ). Сейчас мы введем уравнение НЈВ для задачи (2.6). С этой целью для дважды дифференцируемой функции $f:[0, T] \times \mathbf{R}_{+} \times \mathbf{R} \rightarrow \mathbf{R}$ обозначим через $D f(t, x, y)$ и $D^{2} f(t, x, y)$ следующие векторы частных производных:

$$
\begin{aligned}
D f(t, x, y) & =\left(\frac{\partial}{\partial x} f(t, x, y), \frac{\partial}{\partial y} f(t, x, y)\right)^{\prime}, \\
D^{2} f(t, x, y) & =\left(\frac{\partial^{2}}{\partial x^{2}} f(t, x, y), \frac{\partial^{2}}{\partial y^{2}} f(t, x, y)\right)^{\prime}
\end{aligned}
$$

(здесь штрих обозначает транспонирование). Пусть $\mathbf{q}=\left(q_{1}, q_{2}\right) \in \mathbf{R}^{2}$, $\mathbf{M}=\left(M_{1}, M_{2}\right) \in \mathbf{R}^{2}$ и $\nu=\left(\nu_{1}, \nu_{2}\right) \in \mathbf{R} \times \mathbf{R}_{+}$- некоторые фиксированные параметры. Для этих параметров положим

$H_{0}(x, y, \mathbf{q}, \mathbf{M}, \nu):=\left(r+\nu_{1} \theta(y)-\nu_{2}\right) x q_{1}+\alpha y q_{2}+\frac{1}{2} M_{1} \nu_{1}^{2} x^{2}+\frac{\beta^{2}}{2} M_{2}+\left(\nu_{2} x\right)^{\gamma}$.

Уравнение НЈВ в этом случае имеет следующий вид:

$$
\begin{gathered}
\frac{\partial}{\partial t} z(t, x, y)+\sup _{\nu \in \mathbf{R} \times \mathbf{R}_{+}} H_{0}\left(x, y, D z(t, x, y), D^{2} z(t, x, y), \nu\right)=0, \\
z(T, x, y)=x^{\gamma} .
\end{gathered}
$$

Отметим, что в этом случае для $x>0, q_{1}>0$ и $M_{1}<0$

$\sup _{\nu \in \mathbf{R} \times \mathbf{R}_{+}} H_{0}(x, y, \mathbf{q}, \mathbf{M}, \nu)=x r q_{1}+\alpha y q_{2}+\frac{1}{q_{*}}\left(\frac{\gamma}{q_{1}}\right)^{q_{*}-1}+\frac{\left|\theta(y) q_{1}\right|^{2}}{2\left|M_{1}\right|}+\frac{\beta^{2}}{2} M_{2}$,

где $q_{*}=(1-\gamma)^{-1}$. Для изучения этого уравнения мы представляем $z(t, x, y)$ в виде

$$
z(t, x, y)=x^{\gamma} h(t, y) .
$$

Легко вывести, что функция $h$ удовлетворяет квазилинейному уравнению в частных производных:

$$
\begin{gathered}
\frac{\partial}{\partial t} h(t, y)+Q(y) h(t, y)+\alpha y \frac{\partial}{\partial y} h(t, y) \\
+\frac{\beta^{2}}{2} \frac{\partial^{2}}{\partial y^{2}} h(t, y)+\frac{1}{q_{*}}\left(\frac{1}{h(t, y)}\right)^{q_{*}-1}=0 \\
h(T, y)=1,
\end{gathered}
$$

где

$$
Q(y)=\gamma\left(r+\frac{\theta^{2}(y)}{2(1-\gamma)}\right)
$$


Отметим, что условия на $\sigma(y)$ гарантируют, что функция $Q(y)$ ограничена, дифференцируема и имеет ограниченную производную. Следовательно, мы можем определить

$$
Q_{*}=\sup _{y \in \mathbf{R}} Q(y) \quad \text { и } \quad Q_{1}^{*}=\sup _{y \in \mathbf{R}}\left|\frac{\mathrm{d} Q(y)}{\mathrm{d} y}\right| .
$$

Наша задача заключается в изучении уравнения (3.3). Используя вероятностное представление для линейных уравнений в частных производных (формула Фейнмана-Каца), мы покажем (см. предложение 5.4), что решение этого уравнения является неподвижной точкой для специального оператора интегрального типа, который будет определен в следующем разделе.

4. Необходимые определения. Чтобы изучить уравнение (3.3), прежде всего введем специальное функциональное пространство. Пусть $\mathscr{X}$ - множество равномерно непрерывных функций на $\mathscr{K}:=\left[T_{0}, T\right] \times \mathbf{R}$ со значениями в $[1, \infty)$ таких, что

$$
\|f\|_{\infty}=\sup _{(t, y) \in \mathscr{K}}|f(t, y)| \leqslant \mathbf{r}^{*}
$$

где $\mathbf{r}^{*}=(\widetilde{T}+1) e^{Q_{*} \widetilde{T}}$ и $\widetilde{T}=T-T_{0}$. Определим метрику $\varrho_{*}(\cdot, \cdot)$ в $\mathscr{X}$ следующим образом: для любых $f, g \in \mathscr{X}$ положим

$$
\varrho_{*}(f, g)=\|f-g\|_{*}=\sup _{(t, y) \in \mathscr{K}} e^{-\varkappa(T-t)}|f(t, y)-g(t, y)| .
$$

Здесь $\varkappa=Q_{*}+\zeta+1$ и $\zeta-$ некоторый положительный параметр, значение которого будет уточнено позже. Определим процесс $\eta$ формулой

$$
\mathrm{d} \eta_{s}=\alpha \eta_{s} \mathrm{~d} s+\beta \mathrm{d} \tilde{\mathbf{U}}_{s}, \quad \eta_{0}=Y_{0},
$$

где $\left(\widetilde{\mathbf{U}}_{t}\right)_{t \geqslant 0}-$ стандартное броуновское движение, независимое от $\left(\mathbf{U}_{t}\right)_{t \geqslant 0}$. Таким образом, $\left(\eta_{t}\right)_{t \geqslant 0}$ имеет такое же распределение, как $\left(Y_{t}\right)_{t \geqslant 0}$. Определим отображение Фейнмана-Каца $\mathscr{L}: \mathscr{X} \rightarrow \mathscr{X}$ равенством

$$
\mathscr{L}_{f}(t, y)=\mathbf{E} \mathscr{G}(t, T, y)+\frac{1}{q_{*}} \int_{t}^{T} \mathscr{H}_{f}(t, s, y) \mathrm{d} s,
$$

где $\mathscr{G}(t, s, y)=\exp \left\{\int_{t}^{s} Q\left(\eta_{u}^{t, y}\right) \mathrm{d} u\right\}$,

$$
\mathscr{H}_{f}(t, s, y)=\mathbf{E}\left[\left(f\left(s, \eta_{s}^{t, y}\right)\right)^{1-q_{*}} \mathscr{G}(t, s, y)\right]
$$

и $\left(\eta_{s}^{t, y}\right)_{t \leqslant s \leqslant T}$ - процесс $\eta$ с условием $\eta_{t}=y$. Чтобы решить уравнение HJB, нам нужно найти неподвижную точку отображения $\mathscr{L}$ в $\mathscr{X}$, т.е. функцию $h$ такую, что

$$
h=\mathscr{L}_{h} .
$$


Для этого мы строим следующую итеративную схему. Мы определяем последовательность $h_{0} \equiv 1$,

$$
h_{n}(t, y)=\mathscr{L}_{h_{n-1}}(t, y), \quad n \geqslant 1
$$

и изучаем сходимость этой последовательности в $\mathscr{K}$. На самом деле мы будем использовать существование неподвижной точки для сжимающего оператора в полном метрическом пространстве.

5. Решение уравнения НJВ. В этом разделе мы приводим результат о существовании и единственности решения уравнения (3.3). Для этого мы сначала изучим некоторые свойства оператора ФейнманаКаца $\mathscr{L}$.

Предложение 5.1. Oператор $\mathscr{L}$. «устойчив»в $\mathscr{X}$, m.e.

$$
\mathscr{L}_{f} \in \mathscr{X} \quad \forall f \in \mathscr{X} .
$$

Д о к а з а т е л ь с т в о. Очевидно, что $\mathscr{L}_{f} \geqslant 1$ для любых $f \in \mathscr{X}$. Более того, полагая

$$
\widetilde{f}_{s}=f\left(s, \eta_{s}^{t, y}\right)
$$

мы представим $\mathscr{L}_{f}(t, y)$ в виде

$$
\mathscr{L}_{f}(t, y)=\mathbf{E} \mathscr{G}(t, T, y)+\frac{1}{q_{*}} \int_{t}^{T} \mathbf{E}\left[\left(\tilde{f}_{s}\right)^{1-q_{*}} \mathscr{G}(t, s, y)\right] \mathrm{d} s .
$$

Следовательно, принимая во внимание, что $\tilde{f}_{s} \geqslant 1$ и $q_{*} \geqslant 1$, мы получаем

$$
\mathscr{L}_{f}(t, y) \leqslant e^{Q_{*}(T-t)}+\int_{t}^{T} \frac{1}{q_{*}} e^{Q_{*}(s-t)} \mathrm{d} s \leqslant \mathbf{r}^{*},
$$

где верхняя грань $\mathbf{r}^{*}$ определена в (4.1). Теперь мы должны показать, что $\mathscr{L}_{f}$ - равномерно непрерывная функция на $\mathscr{K}$ для любых $f \in \mathscr{X}$. Для любых $f \in \mathscr{X} \cap C^{1,1}(\mathscr{K})$ рассмотрим уравнение (3.3), т.е.

$$
\begin{gathered}
\frac{\partial}{\partial t} u(t, y)+Q(y) u(t, y)+\alpha y \frac{\partial}{\partial y} u(t, y) \\
+\frac{\beta^{2}}{2} \frac{\partial^{2}}{\partial y^{2}} u(t, y)+\frac{1}{q_{*}}\left(\frac{1}{f(t, y)}\right)^{q_{*}-1}=0 \\
u(T, y)=1 .
\end{gathered}
$$

Полагая здесь $\widetilde{u}(t, y)=u\left(T_{0}+T-t, y\right)$, мы получаем равномерно параболическое уравнение для $\widetilde{u}$ с начальным условием $\widetilde{u}\left(T_{0}, y\right)=1$. Более того, мы знаем, что у $Q$ производная ограничена. Мы заключаем, что 
для любой $f \in \mathscr{X} \cap C^{1,1}(\mathscr{K})$ теорема 5.1 из [17] с $0<l<1$ гарантирует существование единственного решения уравнения (5.4), принадлежащего $C^{1,2}(\mathscr{K})$. Применяя формулу Ито к процессу

$$
\left(u\left(s, \eta_{s}^{t, y}\right) \exp \left\{\int_{t}^{s} Q\left(\eta_{v}^{t, y}\right) \mathrm{d} v\right\}\right)_{t \leqslant s \leqslant T}
$$

и принимая во внимание уравнение (5.4), мы получаем

$$
u(t, y)=\mathscr{L}_{f}(t, y)
$$

Следовательно, $\mathscr{L}_{f}(t, y) \in C^{1,2}(\mathscr{K})$, т.е. $\mathscr{L}_{f} \in \mathscr{X}$ для всех $f \in \mathscr{X} \cap$ $C^{1,1}(\mathscr{K})$. Кроме того, для любой $f \in \mathscr{X}$ существует последовательность $\left(f_{n}\right)_{n \geqslant 1} \in \mathscr{X} \cap C^{1,1}(\mathscr{K})$ такая, что

$$
\sup _{(t, y) \in \mathscr{K}}\left|f_{n}(t, y)-f(t, y)\right| \rightarrow 0, \quad n \rightarrow \infty
$$

Отсюда следует, что

$$
\sup _{(t, y) \in \mathscr{K}}\left|\mathscr{L}_{f_{n}}(t, y)-\mathscr{L}_{f}(t, y)\right| \rightarrow 0, \quad n \rightarrow \infty .
$$

Таким образом, $\mathscr{L}_{f}(t, y)$ равномерно непрерывна на $\mathscr{K}$, т.е. $\mathscr{L}_{f} \in \mathscr{X}$. Предложение 5.1 доказано.

Предложение 5.2. Отображение $\mathscr{L}$ является сжимающим в метрическом пространстве $\left(\mathscr{X}, \varrho_{*}\right)$, т.е. для любых $f, g \in \mathscr{X}$

$$
\varrho_{*}\left(\mathscr{L}_{f}, \mathscr{L}_{g}\right) \leqslant \lambda \varrho_{*}(f, g),
$$

где $\lambda=1 /(\zeta+1) u \zeta>0$.

На самом деле, как будет показано в следствии 6.1, при соответствующем выборе $\zeta$ неравенство (5.6) дает супергеометрическую скорость сходимости последовательности $\left(h_{n}\right)_{n \geqslant 1}$, определенной в $(4.7)$, к предельной функции $h(t, y)$, которая является неподвижной точкой оператора $\mathscr{L}$.

Д о к а з а т е л с т в о. Заметим, что для любых $a>b \geqslant 1$ и $q>0$

$$
b^{-q}-a^{-q} \leqslant q(b-a) .
$$

Используя эту оценку, можно получить, что для любых $f, g \in \mathscr{X}$ и любого $y \in \mathbf{R}$

$$
\begin{aligned}
\left|\mathscr{L}_{f}(t, y)-\mathscr{L}_{g}(t, y)\right| & \leqslant \frac{1}{q_{*}} \mathbf{E} \int_{t}^{T} \mathscr{G}(t, s, y)\left|\left(\widetilde{f}_{s}\right)^{1-q_{*}}-\left(\widetilde{g}_{s}\right)^{1-q_{*}}\right| \mathrm{d} s \\
& \leqslant \gamma \mathbf{E} \int_{t}^{T} \mathscr{G}(t, s, y)\left|\widetilde{f}_{s}-\widetilde{g}_{s}\right| \mathrm{d} s .
\end{aligned}
$$


Напомним, что $\tilde{f}_{s}=f\left(s, \eta_{s}^{t, y}\right)$ и $\widetilde{g}_{s}=g\left(s, \eta_{s}^{t, y}\right)$. Учитывая, что $\mathscr{G}(t, s, y) \leqslant e^{Q_{*}(s-t)}$, мы получаем

$$
\left|\mathscr{L}_{f}(t, y)-\mathscr{L}_{g}(t, y)\right| \leqslant \int_{t}^{T} e^{Q_{*}(s-t)} \mathbf{E}\left|\widetilde{f}_{s}-\widetilde{g}_{s}\right| \mathrm{d} s
$$

Принимая во внимание последнее неравенство, приходим к оценке

$$
\left|\widetilde{f}_{s}-\widetilde{g}_{s}\right| \leqslant e^{\varkappa(T-s)} \varrho_{*}(f, g) \quad \text { п.н. }
$$

Следовательно, для всех $(t, y)$ из $\mathscr{K}$

$$
\left|e^{-\varkappa(T-t)}\left(\mathscr{L}_{f}(t, y)-\mathscr{L}_{g}(t, y)\right)\right| \leqslant \frac{1}{\varkappa-Q_{*}} \varrho_{*}(f, g) .
$$

Используя определение $\varkappa$ (см. (4.2)), мы получим неравенство (5.6). Предложение 5.2 доказано.

Предложение 5.3. Уравнение $\mathscr{L}_{h}=h$ имеет единственное решение в $\mathscr{X}$.

Д о к а з а т е л ь с т в о. Используя то, что оператор $\mathscr{L}$ является сжимающим в $\mathscr{X}$, а также определение (4.7) последовательности $\left(h_{n}\right)_{n \geqslant 1}$, мы получаем, что для любого $n \geqslant 1$

$$
\varrho_{*}\left(h_{n}, h_{n-1}\right) \leqslant \lambda^{n-1} \varrho_{*}\left(h_{1}, h_{0}\right),
$$

т.е. последовательность $\left(h_{n}\right)_{n \geqslant 1}$ является фундаментальной в $\left(\mathscr{X}, \varrho_{*}\right)$. Метрическое пространство $\left(\mathscr{X}, \varrho_{*}\right)$ является полным, так как оно входит в банахово пространство $C^{0,0}(\mathscr{K})$ и норма $\|\cdot\|_{\infty}$ эквивалентна норме $\|\cdot\|_{*}$, определенной в (4.2). Таким образом, последовательность $\left(h_{n}\right)_{n \geqslant 1}$ имеет предел в $\mathscr{X}$, т.е. существует функция $h$ из $\mathscr{X}$, для которой

$$
\lim _{n \rightarrow \infty} \varrho_{*}\left(h, h_{n}\right)=0
$$

Более того, принимая во внимание равенство $h_{n}=\mathscr{L}_{h_{n-1}}$, мы получаем, что для любых $n \geqslant 1$

$$
\varrho_{*}\left(h, \mathscr{L}_{h}\right) \leqslant \varrho_{*}\left(h, h_{n}\right)+\varrho_{*}\left(\mathscr{L}_{h_{n-1}}, \mathscr{L}_{h}\right) \leqslant \varrho_{*}\left(h, h_{n}\right)+\lambda \varrho_{*}\left(h, h_{n-1}\right) .
$$

Последнее выражение стремится к нулю при $n \rightarrow \infty$. Следовательно, $\varrho_{*}\left(h, \mathscr{L}_{h}\right)=0$, т.е. $h=\mathscr{L}_{h}$. Из предложения 5.2 сразу получаем, что решение единственно. Предложение 5.3 доказано.

Мы готовы изложить результат о решении уравнения НЈВ.

Предложение 5.4. Уравнение (3.3) имеет единственное решение $h$, которое является неподвижной точкой оператора $\mathscr{L}: \mathscr{L}_{h}=h$. 
Д о к а з а т е л ь с т в о. Заметим, что в силу леммы 10.5 функция $\mathscr{L}_{h}(t, y)$ является $1 / 2$-гёльдеровской по $t$ на $\{|y|<n\}$ для любых $n \geqslant 1$. Следовательно, выбирая в уравнении (5.4) в качестве $f$ функцию $f_{n}(t, y)=u\left(t, \widetilde{y}_{n}\right)$ (где $\widetilde{y}_{n}$ - проекция $y$ в $\left.[-n, n]\right)$, мы получаем по теореме 5.1 из [17] и лемме 10.5, что это уравнение имеет единственное решение $u_{n}(t, y)$. Понятно, что функция

$$
u(t, y)=\sum_{n \geqslant 1} u_{n}(t, y) \mathbf{1}_{\{n-1<|y| \leqslant n\}}
$$

является решением уравнения (5.4) для $f=u(t, y)$. Принимая во внимание представление $(5.5)$ и уравнение $\mathscr{L}_{h}=h$, мы получаем, что решением уравнения (5.4) является функция

$$
u=\mathscr{L}_{h}=h .
$$

Следовательно, $h$ удовлетворяет уравнению (3.3). Кроме того, это решение единственно, так как $h$ является единственным решением проблемы о неподвижной точке. Предложение 5.4 доказано.

3 а м е ч а н и е 5.1. В [21] можно найти доказательство существования и единственности для более общего квазилинейного уравнения, но это доказательство не дает способа расчета решения, в то время как в нашем случае решение - это неподвижная точка оператора ФейнманаКаца. Кроме того, наш метод позволяет получить супергеометрическую скорость сходимости для последовательности функций, аппроксимирующей решение. Это является очень важным свойством на практике. В работе [3] теорема существования и единственности доказывается с помощью соответствующей локальной теоремы. Следует также отметить, что метод применения сжимающих отображений или теоремы о неподвижной точке для изучения нелинейных уравнений в частных производных уже встречался ранее в других постановках (см., например, [8] и [24]). Термин «обобщенное решение» используется для квазилинейных/полулинейных уравнений в частных производных, и решение представляется как неподвижная точка для оператора Фейнмана-Каца.

6. Супергеометрическая скорость сходимости. Для последовательности $\left(h_{n}\right)_{n \geqslant 1}$, определенной в $(4.7)$, и решения уравнения $\mathscr{L}_{h}=h$ мы изучим отклонение

$$
\Delta_{n}(t, y)=h(t, y)-h_{n}(t, y)
$$

В следующей теореме мы выводим верхнюю границу для модуля этой разности, что позволит выбрать $\zeta$ для параметра сжатия $\lambda$ так, чтобы получить супергеометрическую скорость сходимости для последовательности $\left(h_{n}\right)_{n \geqslant 1}$. 
Теорема 6.1. Уравнение $\mathscr{L}_{h}=h$ имеет единственное решение $h$ в $\mathscr{X}$ такое, что для всех $n \geqslant 1 u \zeta>0$

$$
\sup _{(t, y) \in \mathscr{K}}\left|\Delta_{n}(t, y)\right| \leqslant \mathbf{B}^{*} \lambda^{n}
$$

где $\mathbf{B}^{*}=e^{\varkappa \widetilde{T}}\left(1+\mathbf{r}^{*}\right) /(1-\lambda), \widetilde{T}=T-T_{0}$ иконстанта и введена в (4.2).

Д о к а з а т е л ь с т в о. Единственность установлена в предложении 5.3. Далее, из (5.9) легко видеть, что для любого $n \geqslant 1$

$$
\varrho_{*}\left(h, h_{n}\right) \leqslant \frac{\lambda^{n}}{1-\lambda} \varrho_{*}\left(h_{1}, h_{0}\right) .
$$

В силу предложения 5.1 все функции $h_{n}$ принадлежат $\mathscr{X}$, и, значит, по определению пространства $\mathscr{X}$

$$
\varrho_{*}\left(h_{1}, h_{0}\right) \leqslant \sup _{(t, y) \in \mathscr{K}}\left|h_{1}(t, y)-1\right| \leqslant 1+\mathbf{r}^{*} .
$$

Учитывая, что $\sup _{(t, y) \in \mathscr{K}}\left|\Delta_{n}(t, y)\right| \leqslant e^{\varkappa \widetilde{T}} \varrho_{*}\left(h, h_{n}\right)$, мы получаем неравенство (6.1). Теорема 6.1 доказана.

Теперь мы можем минимизировать верхнюю границу (6.1) по $\zeta>0$. Действительно,

$$
\mathbf{B}^{*} \lambda^{n}=\mathbf{C}^{*} \exp \left\{\mathbf{g}_{n}(\zeta)\right\},
$$

где $\mathbf{C}^{*}=\left(1+\mathbf{r}^{*}\right) e^{\left(Q_{*}+1\right) \widetilde{T}}$ и функция $\mathbf{g}_{n}$ имеет вид

$$
\mathbf{g}_{n}(x)=x \widetilde{T}-\ln x-(n-1) \ln (1+x) .
$$

Минимизируем эту функцию по $x>0$ :

$$
\min _{x>0} \mathbf{g}_{n}(x)=x_{n}^{*} \widetilde{T}-\ln x_{n}^{*}-(n-1) \ln \left(1+x_{n}^{*}\right),
$$

где

$$
x_{n}^{*}=\frac{\sqrt{(\widetilde{T}-n)^{2}+4 \widetilde{T}}+n-\widetilde{T}}{2 \widetilde{T}} .
$$

Следовательно, при $\zeta=\zeta_{n}^{*}=x_{n}^{*}$ мы получаем оптимальную границу (6.1).

Следствие 6.1. Уравнение $\mathscr{L}_{h}=h$ имеет единственное решение $h$ в $\mathscr{X}$ такое, что для всех $n \geqslant 1$

$$
\sup _{(t, y) \in \mathscr{K}}\left|\Delta_{n}(t, y)\right| \leqslant \mathbf{U}_{n}^{*},
$$

где $\mathbf{U}_{n}^{*}=\mathbf{C}^{*} \exp \left\{\mathbf{g}_{n}^{*}\right\}$. Более того, для любого $0<\delta<1$

$$
\mathbf{U}_{n}^{*}=O\left(n^{-\delta n}\right), \quad n \rightarrow \infty .
$$

Это означает, что скорость сходимости бъстрее любой геометрической, т.е. является супергеометрической. 
7. Известные параметры. Сейчас мы рассмотрим нашу задачу оптимального потребления и инвестирования в случае рынков с известными параметрами. Следующая теорема является аналогом теоремы 3.4 в [1]. Основное различие заключается в том, что коэффициент сноса у процесса $Y$ в [1] должен быть ограниченным, что не позволяет использовать процесс Орнштейна-Уленбека.

Теорема 7.1. Оптимальное значение $J\left(T_{0}, x, y, \vartheta\right)$ для оптимизаиионной проблемь (2.6) дается формулой

$$
J_{T_{0}}^{*}=J\left(T_{0}, x, y, \vartheta^{*}\right)=\sup _{\vartheta \in \mathscr{V}} J\left(T_{0}, x, y, \vartheta\right)=x^{\gamma} h\left(T_{0}, y\right),
$$

где $h(t, y)$ - единственное решение уравнения (3.3). Кроме того, для всех $T_{0} \leqslant t \leqslant T$ оптимальная финансовая стратегия $\vartheta^{*}=\left(\pi^{*}, c^{*}\right)$ имеет вид

$$
\pi_{t}^{*}=\pi^{*}\left(Y_{t}\right)=\frac{\theta\left(Y_{t}\right)}{1-\gamma}, \quad c_{t}^{*}=c^{*}\left(t, Y_{t}\right)=\left(h\left(t, Y_{t}\right)\right)^{-q_{*}} .
$$

Оптимальньй процесс капитала $\left(X_{t}^{*}\right)_{T_{0} \leqslant t \leqslant T}$ удовлетворяет следующему стохастическому дифференииальному уравнению:

$$
\mathrm{d} X_{t}^{*}=\mathbf{a}^{*}\left(t, Y_{t}\right) X_{t}^{*} \mathrm{~d} t+X_{t}^{*} \mathbf{b}^{*}\left(Y_{t}\right) \mathrm{d} W_{t}, \quad X_{T_{0}}^{*}=x
$$

¿əe

$$
\mathbf{a}^{*}(t, y)=\frac{|\theta(y)|^{2}}{1-\gamma}+r-(h(t, y))^{-q_{*}}, \quad \mathbf{b}^{*}(y)=\frac{\theta(y)}{1-\gamma} .
$$

Решение $X_{t}^{*}$ может быть записано в виде

$$
X_{s}^{*}=X_{t}^{*} \exp \left\{\int_{t}^{s} \mathbf{a}^{*}\left(v, Y_{v}\right) \mathrm{d} v\right\} \mathscr{E}_{t, s}
$$

где $\mathscr{E}_{t, s}=\exp \left\{\int_{t}^{s} \mathbf{b}^{*}\left(Y_{v}\right) \mathrm{d} W_{v}-(1 / 2) \int_{t}^{s}\left|\mathbf{b}^{*}\left(Y_{v}\right)\right|^{2} \mathrm{~d} v\right\}$.

Доказательство теоремы такое же, как доказательство теоремы 3.4 в [1], и поэтому опускается. Подчеркнем только, что теорема доказывается с помощью проверочной теоремы (теорема 6.2 в [1]) для задачи (2.6), из которой, в частности, вытекает, что решение уравнения (3.1) является единственным в соответствующем функциональном классе.

8. Неизвестные параметры. В этом разделе мы рассмотрим рынок Блэка-Шоулса с неизвестным параметром $\alpha$ (случай известного параметра $\mu$ изучается в п. 8.2, случай, когда $\mu$ не известен, - в п. 8.3). Мы наблюдаем процесс $Y$ в интервале $\left[0, T_{0}\right]$ (в этом случае $T_{0}>0$ ) и используем последовательные методы для оценки сноса. После этого мы решаем задачу оптимального потребления и инвестирования на конечном интервале $\left[T_{0}, T\right]$ и изучаем поведение оптимального значения целевой функции $J^{*}\left(T_{0}, x, y\right)$. Определим $\widehat{J}_{T_{0}}^{*}$ как оценку для $J_{T_{0}}^{*}$ :

$$
\widehat{J}_{T_{0}}^{*}:=\mathbf{E}_{T_{0}}\left(\int_{T_{0}}^{T}\left(\widehat{c}_{t}^{*}\right)^{\gamma}\left(\widehat{X}_{t}^{*}\right)^{\gamma} \mathrm{d} t+\left(\widehat{X}_{T}^{*}\right)^{\gamma}\right)
$$


где $\mathbf{E}_{T_{0}}$ - условное математическое ожидание $\mathbf{E}\left(\cdot \mid \mathscr{F}_{T_{0}}\right)$ и для упрощения обозначений мы пишем $\widehat{X}_{t}^{*}$ вместо $X_{t}^{\widehat{\vartheta}_{*}}$. Из (7.4) получаем

$$
\widehat{X}_{s}^{*}=\widehat{X}_{t}^{*} \exp \left\{\int_{t}^{s} \widehat{\mathbf{a}}^{*}\left(v, Y_{v}\right) \mathrm{d} v\right\} \widehat{\mathscr{E}}_{t, s},
$$

где $\widehat{\mathscr{E}}_{t, s}=\exp \left\{\int_{t}^{s} \widehat{\mathbf{b}}^{*}\left(Y_{v}\right) \mathrm{d} W_{v}-(1 / 2) \int_{t}^{s}\left|\widehat{\mathbf{b}}^{*}\left(Y_{v}\right)\right|^{2} \mathrm{~d} v\right\}$. Функции $\mathbf{a}^{*}(t, y)$ и $\mathbf{b}^{*}(t, y)$ определяются формулами

$$
\widehat{\mathbf{a}}^{*}(t, y)=\frac{|\widehat{\theta}(y)|^{2}}{1-\gamma}+r-(\widehat{h}(t, y))^{-q_{*}}, \quad \widehat{\mathbf{b}}^{*}(y)=\frac{\widehat{\theta}(y)}{1-\gamma}, \quad \widehat{\theta}(y)=\frac{\widehat{\mu}-r}{\sigma(y)} .
$$

Оцениваемый процесс потребления - это $\widehat{c}_{t}^{*}=\widehat{c}^{*}\left(t, Y_{t}\right)=\left(\widehat{h}\left(t, Y_{t}\right)\right)^{-q_{*}}$ где $\widehat{h}(t, y)$ - единственное решение уравнения $\widehat{\mathscr{L}}_{h}=h$. Оператор $\widehat{\mathscr{L}}$ определен следующим образом:

$$
\widehat{\mathscr{L}}_{f}(t, y)=\mathbf{E} \widehat{\mathscr{G}}(t, T, y)+\frac{1}{q_{*}} \int_{t}^{T} \mathbf{E}\left(\left[f\left(s, \widehat{\eta}_{s}^{t, y}\right)\right]^{1-q_{*}} \widehat{\mathscr{G}}(t, s, y)\right) \mathrm{d} s,
$$

где $\widehat{\mathscr{G}}(t, s, y)=\exp \left\{\int_{t}^{s} \widehat{Q}\left(\widehat{\eta}_{u}^{t, y}\right) \mathrm{d} u\right\}$ и

$$
\mathrm{d} \widehat{\eta}_{s}^{t, y}=\widehat{\alpha} \widehat{\eta}_{s}^{t, y} \mathrm{~d} s+\beta \mathrm{d} \widetilde{\mathbf{U}}_{s}, \quad \widehat{\eta}_{t}^{t, y}=y .
$$

Здесь $\widehat{\alpha}$ и $\widehat{\mu}$ - некоторые оценки для $\alpha$ и $\mu$, которые будут определены позже.

8.1. Последовательная процедура. Как уже отмечалось выше, в случае неизвестного параметра $\alpha$ мы предполагаем, что он принимает значения в некотором ограниченном интервале $\left[\alpha_{2}, \alpha_{1}\right]$, где $\alpha_{2} \leqslant \alpha \leqslant$ $\alpha_{1}<0$. Оценку $\widehat{\alpha}$ мы определим как проекцию на интервал $\left[\alpha_{2}, \alpha_{1}\right]$ последовательной оценки $\alpha^{*}$, т.е.

$$
\widehat{\alpha}=\operatorname{Proj}_{\left[\alpha_{2}, \alpha_{1}\right]} \alpha^{*}, \quad \alpha^{*}=\frac{\int_{0}^{\tau_{H}} Y_{t} \mathrm{~d} Y_{t}}{H} \mathbf{1}_{\left\{\tau_{H} \leqslant T_{0}\right\}},
$$

где $\tau_{H}=\inf \left\{t \geqslant 0: \int_{0}^{t} Y_{s}^{2} \mathrm{~d} s \geqslant H\right\}$ и для любого $x \in \mathbf{R}$

$$
\operatorname{Proj}_{\left[\alpha_{2}, \alpha_{1}\right]}(x)=\alpha_{2} \mathbf{1}_{\left\{x \leqslant \alpha_{2}\right\}}+x \mathbf{1}_{\left\{\alpha_{2} \leqslant x \leqslant \alpha_{1}\right\}}+\alpha_{1} \mathbf{1}_{\left\{x \geqslant \alpha_{1}\right\}} .
$$

Далее, определим функцию $\epsilon(\cdot)$, которая будет использоваться позже при доказательстве $\delta$-оптимальности:

$$
\epsilon\left(T_{0}\right)=\sqrt{\frac{\beta^{2}}{\beta_{2}\left(T_{0}-T_{0}^{5 / 6}\right)}+\frac{\alpha_{2}^{2}}{\beta^{12}}\left(\frac{\mathbf{k}(3)}{T_{0}^{2}}\right)},
$$

где $\beta_{2}=\beta^{2} /\left(2\left|\alpha_{2}\right|\right), \mathbf{k}(m)=3^{2 m-1}\left(Y_{0}^{2 m}+\left[1+(m(2 m-1))^{m}(2 \beta)^{2 m}\right] \mathbf{k}_{1}(m)\right)$,

$$
\mathbf{k}_{1}(m)=2^{2 m-1}\left(Y_{0}^{2 m}+(2 m-1) ! ! \beta_{1}^{m}\right) \quad \text { и } \quad \beta_{1}=\frac{\beta^{2}}{2\left|\alpha_{1}\right|} .
$$

Следующее предложение дает верхнюю границу для математического ожидания $\mathbf{E}|\bar{\alpha}|$ отклонения $\bar{\alpha}=\widehat{\alpha}-\alpha$. 
Предложение 8.1. Для любых $0<T_{0}<T$ последовательная прочедура (8.6) удовлетворяет следующему неравенству:

$$
\sup _{\alpha_{2} \leqslant \alpha \leqslant \alpha_{1}} \mathbf{E}|\bar{\alpha}| \leqslant \epsilon\left(T_{0}\right) .
$$

Д о к а з а т е л ь с т в о. Заметим, что $\mathbf{E}|\bar{\alpha}| \leqslant \mathbf{E}\left|\alpha^{*}-\alpha\right|$, поэтому достаточно доказать неравенство $\sup _{\alpha_{2} \leqslant \alpha \leqslant \alpha_{1}} \mathbf{E}\left|\alpha^{*}-\alpha\right| \leqslant \epsilon\left(T_{0}\right)$. Кроме того, мы знаем из $[19$, гл. 17$]$, что оценка максимального правдоподобия для $\alpha$ равна

$$
\frac{\int_{0}^{T_{0}} Y_{t} \mathrm{~d} Y_{t}}{\int_{0}^{T_{0}} Y_{t}^{2} \mathrm{~d} t}
$$

В качестве оценки параметра $\alpha$ мы используем последовательную оценку максимального правдоподобия, предложенную в [19] и [23]:

$$
\widetilde{\alpha}=\frac{\int_{0}^{\tau_{H}} Y_{t} \mathrm{~d} Y_{t}}{\int_{0}^{\tau_{H}} Y_{t}^{2} \mathrm{~d} t}=\alpha+\beta \frac{\int_{0}^{\tau_{H}} Y_{t} \mathrm{~d} \mathbf{U}_{t}}{H} .
$$

Учитывая, что $\int_{0}^{\infty} Y_{t}^{2} \mathrm{~d} t=+\infty$ п.н., получаем, что $\widetilde{\alpha} \sim \mathscr{N}\left(\alpha, \beta^{2} / H\right)$ и, следовательно,

$$
\mathbf{E}|\widetilde{\alpha}-\alpha|^{2}=\frac{\beta^{2}}{H} .
$$

Проблема с использованием этой оценки состоит в том, что $\tau_{H}$ может быть больше, чем $T_{0}$. Чтобы преодолеть эту трудность, мы используем усеченную модификацию последовательной процедуры $\widehat{\alpha}$ из [14], т.е. $\alpha^{*}=$ $\widetilde{\alpha} \mathbf{1}_{\left\{\tau_{H} \leqslant T_{0}\right\}}$. Заметим, что

$$
\begin{aligned}
\alpha^{*}-\alpha & =\left(\alpha^{*}-\alpha\right) \mathbf{1}_{\left\{\tau_{H} \leqslant T_{0}\right\}}+\left(\alpha^{*}-\alpha\right) \mathbf{1}_{\left\{\tau_{H}>T_{0}\right\}} \\
& =\beta \frac{\int_{0}^{\tau_{H}} Y_{t} \mathrm{~d} \mathbf{U}_{t}}{H} \mathbf{1}_{\left\{\tau_{H} \leqslant T_{0}\right\}}-\alpha \mathbf{1}_{\left\{\tau_{H}>T_{0}\right\}} .
\end{aligned}
$$

Поэтому

$$
\begin{aligned}
\mathbf{E}\left(\alpha^{*}-\alpha\right)^{2} & =\frac{\beta^{2}}{H^{2}} \mathbf{E}\left(\int_{0}^{\tau_{H}} Y_{t} \mathrm{~d} \mathbf{U}_{t} \mathbf{1}_{\left\{\tau_{H} \leqslant T_{0}\right\}}\right)^{2}+\alpha^{2} \mathbf{P}\left(\tau_{H}>T_{0}\right) \\
& \leqslant \frac{\beta^{2}}{H^{2}} \mathbf{E}\left(\int_{0}^{\tau_{H}} Y_{t} \mathrm{~d} \mathbf{U}_{t}\right)^{2}+\alpha^{2} \mathbf{P}\left(\tau_{H}>T_{0}\right) \\
& \leqslant \frac{\beta^{2}}{H}+\alpha^{2} \mathbf{P}\left(\int_{0}^{T_{0}} Y_{t}^{2} \mathrm{~d} t<H\right) .
\end{aligned}
$$

Кроме того, по формуле Ито

$$
\mathrm{d} Y_{t}^{2}=2 Y_{t} \mathrm{~d} Y_{t}+\beta^{2} \mathrm{~d} t=\left(2 \alpha Y_{t}^{2}+\beta^{2}\right) \mathrm{d} t+2 \beta Y_{t} \mathrm{~d} \mathbf{U}_{t} .
$$

Отсюда следует равенство

$$
\int_{0}^{T_{0}}\left(2 \alpha Y_{t}^{2}+\beta^{2}\right) \mathrm{d} t=Y_{T_{0}}^{2}-Y_{0}^{2}-2 \beta \int_{0}^{T_{0}} Y_{t} \mathrm{~d} \mathbf{U}_{t}
$$


Учитывая, что $\alpha_{2} \leqslant \alpha \leqslant \alpha_{1}<0$, и используя неравенство Маркова, мы получаем для любого целого $m>0$

$$
\begin{aligned}
\mathbf{P}\left(\int_{0}^{T_{0}} Y_{t}^{2} \mathrm{~d} t<H\right) & =\mathbf{P}\left(\int_{0}^{T_{0}}\left(2 \alpha Y_{t}^{2}+\beta^{2}\right) \mathrm{d} t>2 \alpha H+\beta^{2} T_{0}\right) \\
& =\mathbf{P}\left(Y_{T_{0}}^{2}-Y_{0}^{2}-2 \beta \int_{0}^{T_{0}} Y_{t} \mathrm{~d} \mathbf{U}_{t}>2 \alpha H+\beta^{2} T_{0}\right) \\
& \leqslant \frac{\mathbf{E}\left(Y_{T_{0}}^{2}-Y_{0}^{2}-2 \beta \int_{0}^{T_{0}} Y_{t} \mathrm{~d} \mathbf{U}_{t}\right)^{2 m}}{\left(2 \alpha_{2} H+\beta^{2} T_{0}\right)^{2 m}} .
\end{aligned}
$$

Здесь $2 \alpha H+\beta^{2} T_{0}>0$, т.е. $0<H<\beta_{2} T_{0}$. Для стохастического интеграла $\xi_{t}=\int_{0}^{t} \beta e^{\alpha(t-v)} \mathrm{d} \mathbf{U}_{v}$ мы получаем при любом $m \in \mathbf{N}_{*}$ оценку

$$
\mathbf{E} \xi_{t}^{2 m}=(2 m-1) ! !\left[\mathbf{E} \xi_{t}^{2}\right]^{m} \leqslant(2 m-1) ! ! \beta_{1}^{m} .
$$

Более того, так как $Y_{T_{0}}=Y_{0} e^{\alpha T_{0}}+\xi_{T_{0}}$, то

$$
\mathbf{E} Y_{T_{0}}^{2 m} \leqslant 2^{2 m-1}\left(\mathbf{E}\left(Y_{0} e^{\alpha T_{0}}\right)^{2 m}+\mathbf{E} \xi_{T_{0}}^{2 m}\right) \leqslant \mathbf{k}_{1}(m) .
$$

Кроме того (см., например, [18, лемма 4.12]),

$$
\mathbf{E}\left(\int_{0}^{T_{0}} Y_{t} \mathrm{~d} \mathbf{U}_{t}\right)^{2 m} \leqslant(m(2 m-1))^{m} T_{0}^{m-1} \int_{0}^{T_{0}} \mathbf{E} Y_{s}^{2 m} \mathrm{~d} s \leqslant \mathbf{k}_{2}(m) T_{0}^{m},
$$

где $\mathbf{k}_{2}(m)=(m(2 m-1))^{m} \mathbf{k}_{1}(m)$. Мы получаем

$$
\mathbf{P}\left(\int_{0}^{T_{0}} Y_{t}^{2} \mathrm{~d} t<H\right) \leqslant \frac{3^{2 m-1}\left(Y_{0}^{2 m}+\mathbf{k}_{1}(m)+(2 \beta)^{2 m} \mathbf{k}_{2}(m) T_{0}^{m}\right)}{\left(2 \alpha_{2} H+\beta^{2} T_{0}\right)^{2 m}} .
$$

Положим $H=\beta_{2}\left(T_{0}-T_{0}^{\varepsilon}\right)$ для некоторого $\varepsilon$. Тогда

$$
\mathbf{P}\left(\int_{0}^{T_{0}} Y_{t}^{2} \mathrm{~d} t<H\right) \leqslant \frac{1}{\left(\beta^{2}\right)^{2 m}} \frac{\mathbf{k}(m)}{T_{0}^{m(2 \varepsilon-1)}} .
$$

Подставляя это неравенство в (8.8), приходим к оценке

$$
\mathbf{E}\left(\alpha^{*}-\alpha\right)^{2} \leqslant \frac{\beta^{2}}{\beta_{2}\left(T_{0}-T_{0}^{\varepsilon}\right)}+\frac{\alpha^{2}}{\beta^{4 m}} \frac{\mathbf{k}(m)}{T_{0}^{m(2 \varepsilon-1)}} .
$$

Выберем $\varepsilon=5 / 6$ и $m=3$, так что $m(2 \varepsilon-1)=2$, что дает $\epsilon^{2}\left(T_{0}\right)$ и, следовательно, желаемый результат. Предложение 8.1 доказано.

8.2. Известный параметр $\mu$. В этом пункте мы рассматриваем задачу оптимального потребления и инвестирования для рынков с известным $\mu$ и неизвестным $\alpha$, т.е. в этом случае $\widehat{\mu}=\mu$ и, следовательно, $\widehat{\theta}(y)=\theta(y)$ в (8.3). Положим

$$
\begin{aligned}
\mathbf{h}_{1} & =\frac{1+2 \gamma+\zeta_{0}}{1+\zeta_{0}} \frac{\widetilde{T}}{\left|\alpha_{1}\right|}\left(2 Q_{1}^{*} \widetilde{T}+\gamma h_{1}^{*}\right), \\
\Gamma & =\left(q_{*} \widetilde{T}(\sqrt{\widetilde{c}})^{\gamma}+(\widetilde{T}+1)\left(\sqrt{\widetilde{c} q_{*}}\right)^{\gamma}\right) \frac{1}{\varkappa^{\gamma}} e^{\gamma \varkappa \widetilde{T}},
\end{aligned}
$$


где $\zeta_{0}>0, \widetilde{c}=4 \widetilde{T} e^{2 c_{0}} \widetilde{T}$,

$$
c_{0}=\theta_{*}^{4}+\theta_{*}^{2}(2|r|+3)+(|r|+1)^{2} \quad \text { и } \quad \theta_{*}=\frac{\mu_{*}+|r|}{\sigma_{1}(1-\gamma)} .
$$

Кроме того,

$$
h_{1}^{*}=\left(\widetilde{T} Q_{1}^{*}+\frac{Q_{1}^{*} \widetilde{T}^{2}}{q_{*}}\right) e^{Q_{*} \widetilde{T}}+\frac{3}{q_{*}} \sqrt{\frac{2\left|\alpha_{2}\right|}{\beta^{2}\left(1-e^{2 \alpha_{2}}\right)}} e^{Q_{*} \widetilde{T}} \widetilde{T} .
$$

Теорема 8.1. Для любьх $0<T_{0}<T$ и любьл $m \geqslant 1$

$$
\sup _{\alpha_{2} \leqslant \alpha \leqslant \alpha_{1}} \sup _{|\mu| \leqslant \mu_{*}} \mathbf{E}\left|\widehat{J}_{T_{0}}^{*}-J^{*}\left(T_{0}, x, Y_{T_{0}}\right)\right| \leqslant \delta_{m},
$$

$2 \partial e$

$$
\delta_{m}=\delta_{m}\left(x, T_{0}\right)=\Gamma \mathbf{h}_{1}^{\gamma} x^{\gamma}\left(\left(2 \iota_{0}\right)^{\gamma}+\left(\frac{(2 m-1) ! ! \beta^{2 m}}{\left(2\left|\alpha_{1}\right|\right)^{m}}\right)^{\gamma /(2 m)}\right) \epsilon^{\gamma}\left(T_{0}\right),
$$

$\iota_{0}=\beta / \sqrt{2\left|\alpha_{1}\right|}$ и функиия $\epsilon(\cdot)$ определена в (8.7).

Д о к а з а т е л ь с т в о. Прежде всего заметим, что

$$
\sup _{(s, y) \in \mathscr{K}}\left(\left|\mathbf{a}^{*}(s, y)\right|^{2}+\left|\mathbf{b}^{*}(y)\right|^{2}\right) \leqslant c_{0} .
$$

Кроме того, для любых $T_{0}<T$

$$
\begin{aligned}
\left|\widehat{J}_{T_{0}}^{*}-J_{T_{0}}^{*}\right| & \leqslant \mathbf{E}_{T_{0}} \int_{T_{0}}^{T}\left|\left(\widehat{c}_{t}^{*}\right)^{\gamma}\left(\widehat{X}_{t}^{*}\right)^{\gamma}-\left(c_{t}^{*}\right)^{\gamma}\left(X_{t}^{*}\right)^{\gamma}\right| \mathrm{d} t+\mathbf{E}_{T_{0}}\left|\left(\widehat{X}_{T}^{*}\right)^{\gamma}-\left(X_{T}^{*}\right)^{\gamma}\right| \\
& \leqslant \mathbf{E}_{T_{0}} \int_{T_{0}}^{T}\left|\widehat{c}_{t}^{*} \widehat{X}_{t}^{*}-c_{t}^{*} X_{t}^{*}\right|^{\gamma} \mathrm{d} t+\mathbf{E}_{T_{0}}\left|\widehat{X}_{T}^{*}-X_{T}^{*}\right|^{\gamma}
\end{aligned}
$$

Используя приводимую ниже лемму 8.1, мы получаем

$$
\left|\widehat{J}_{T_{0}}^{*}-J^{*}\left(T_{0}, x, Y_{T_{0}}\right)\right| \leqslant \Gamma \mathbf{h}_{1}^{\gamma} x^{\gamma}\left(2 \iota_{0}+\left|Y_{T_{0}}\right|\right)^{\gamma}|\bar{\alpha}|^{\gamma}
$$

и, следовательно,

$$
\mathbf{E}\left|\widehat{J}_{T_{0}}^{*}-J^{*}\left(T_{0}, x, Y_{T_{0}}\right)\right| \leqslant \Gamma x^{\gamma} \mathbf{h}_{1}^{\gamma}\left(2 \iota_{0}\right)^{\gamma} \mathbf{E}|\bar{\alpha}|^{\gamma} \Gamma x^{\gamma} \mathbf{h}_{1}^{\gamma} \mathbf{E}\left(\left|Y_{T_{0}}\right|^{\gamma}|\bar{\alpha}|^{\gamma}\right) .
$$

По неравенствам Гёльдера и Йенсена для $m^{\prime}=m(2-\gamma) / \gamma>1$, где $m \geqslant 1$, имеем

$$
\begin{aligned}
\mathbf{E}\left(\left|Y_{T_{0}}\right|^{\gamma}|\bar{\alpha}|^{\gamma}\right) & \leqslant\left(\mathbf{E}\left|Y_{T_{0}}\right|^{2 \gamma /(2-\gamma)}\right)^{(2-\gamma) / 2}\left(\mathbf{E}|\bar{\alpha}|^{2}\right)^{\gamma / 2} \\
& \leqslant\left(\mathbf{E}\left|Y_{T_{0}}\right|^{2 \gamma m^{\prime} /(2-\gamma)}\right)^{(2-\gamma) /\left(2 m^{\prime}\right)} \epsilon^{\gamma}\left(T_{0}\right) \\
& \leqslant\left(\mathbf{E}\left|Y_{T_{0}}\right|^{2 m}\right)^{\gamma /(2 m)} \epsilon^{\gamma}\left(T_{0}\right) .
\end{aligned}
$$


Из [11, лемма 1.1.1] следует, что $\mathbf{E}\left|Y_{T_{0}}\right|^{2 m} \leqslant c_{m}\left(T_{0}\right) \leqslant c_{m}(0)$, где

$$
c_{m}\left(T_{0}\right)=(2 m-1) ! ! \beta^{2 m}\left(\frac{1-e^{2 \alpha T_{0}}}{2|\alpha|}\right)^{m} .
$$

Мы получаем, что для любого $m \geqslant 1$

$$
\mathbf{E}\left(\left|Y_{T_{0}}\right|^{\gamma}|\bar{\alpha}|^{\gamma}\right) \leqslant\left(\frac{(2 m-1) ! ! \beta^{2 m}}{\left(2\left|\alpha_{1}\right|\right)^{m}}\right)^{\gamma /(2 m)} \epsilon^{\gamma}\left(T_{0}\right),
$$

что дает желаемый результат. Теорема 8.1 доказана.

3 а м е ч а н и е 8.1. Математическое ожидание $\mathbf{E}\left|\widehat{J}_{T_{0}}^{*}-J^{*}\left(T_{0}, x, y\right)\right|$ в теореме 8.1 можно сделать произвольно малым либо за счет увеличения интервала наблюдения $\left[0, T_{0}\right]$ процесса $Y$, либо за счет уменьшения начального капитала $x$. Это означает, что даже в случае, когда интервал оценивания недостаточно широк (как часто и бывает на практике), мы всегда можем найти стратегию, которая сколь угодно близка к оптимальной. Но в этом случае следует быть осторожными в выборе первоначального вклада, принимая во внимание оценку (8.11).

Лемма 8.1. Для любых $0<T_{0} \leqslant T$

$$
\mathbf{E}_{T_{0}} \sup _{T_{0} \leqslant s \leqslant T}\left(\widehat{X}_{s}^{*}\right)^{2}<x^{2} \widetilde{c} .
$$

Кроме того,

$$
\sup _{T_{0} \leqslant t \leqslant T} \mathbf{E}_{T_{0}}\left|\widehat{X}_{t}^{*}-X_{t}^{*}\right|^{\gamma} \leqslant k_{1} x^{\gamma}\left(\mathbf{h}_{1}\left(2 \iota_{0}+\left|Y_{T_{0}}\right|\right)\right)^{\gamma}|\bar{\alpha}|^{\gamma}
$$

$u$

$$
\mathbf{E}_{T_{0}} \int_{T_{0}}^{T}\left|\widehat{c}_{t}^{*} \widehat{X}_{t}^{*}-c_{t}^{*} X_{t}^{*}\right|^{\gamma} \mathrm{d} t \leqslant k_{2} x^{\gamma}\left(\mathbf{h}_{1}\left(2 \iota_{0}+\left|Y_{T_{0}}\right|\right)\right)^{\gamma}|\bar{\alpha}|^{\gamma}
$$

zде $k_{1}=\left(\sqrt{\widetilde{c} q_{*}}\right)^{\gamma} e^{\gamma \varkappa \widetilde{T}} / \varkappa^{\gamma} u k_{2}=\left(\widetilde{T}\left(\sqrt{\widetilde{c} q_{*}}\right)^{\gamma}+\widetilde{d}^{\gamma} q_{*} \widetilde{T}\right) e^{\gamma \varkappa \widetilde{T}} / \varkappa^{\gamma}$.

Д о к а з а т е л ь с т в о. Прежде всего заметим, что, так же как в доказательстве теоремы $8.1,\left(A^{*}\right)^{2}+\left(B^{*}\right)^{2} \leqslant c_{0}$, где

$$
A^{*}=\sup _{(s, y) \in \mathscr{K}}\left|\widehat{\mathbf{a}}^{*}(s, y)\right| \quad \text { и } \quad B^{*}=\sup _{y \in \mathbf{R}}\left|\widehat{\mathbf{b}}^{*}(y)\right| \text {. }
$$

Функции $\widehat{\mathbf{a}}^{*}(s, y)$ и $\widehat{\mathbf{b}}^{*}(y)$ определены в (8.3). Далее, из (8.2) понятно, что для ограниченной функции $\widehat{\mathbf{b}}^{*}(y)$ процесс $\left(\widehat{\mathscr{E}}_{t, s}\right)_{t \leqslant s \leqslant T}$ является квадратично интегрируемым мартингалом, и по неравенству Дуба

$$
\begin{aligned}
\mathbf{E}_{T_{0}} \sup _{T_{0} \leqslant s \leqslant T}\left(\widehat{X}_{s}^{*}\right)^{2} & \leqslant x^{2} e^{2 \widetilde{T} A^{*}} \mathbf{E} \sup _{t \leqslant s \leqslant T} \widehat{\mathscr{E}}_{t, s}^{2} \leqslant x^{2} \cdot 4 e^{2 \widetilde{T} A^{*}} \mathbf{E} \widehat{\mathscr{E}}_{t, T}^{2} \\
& \leqslant 4 x^{2} e^{2 \widetilde{T} A^{*}} e^{\widetilde{T}\left(B^{*}\right)^{2}}
\end{aligned}
$$


Это доказывает оценку (8.14). Положим $\Delta_{t}=\widehat{X}_{t}^{*}-X_{t}^{*}, A_{s}=\mathbf{a}^{*}\left(s, Y_{s}\right)$, $B_{s}=\mathbf{b}^{*}\left(Y_{s}\right), \widehat{A}_{s}=\widehat{\mathbf{a}}^{*}\left(s, Y_{s}\right)$ и $\widehat{B}_{s}=\widehat{\mathbf{b}}^{*}\left(Y_{s}\right)$. Ясно, что если $\mu$ известно (т.е. $\widehat{\mu}=\mu$ ), то функция $\widehat{B}_{s}$ совпадает с $B_{s}$. Но мы сохраним обозначение $\widetilde{B}_{s}$, чтобы использовать его в случае неизвестного параметра $\mu$. Далее, положим $\varphi_{1}(s)=\widehat{A}_{s} \widehat{X}_{s}^{*}-A_{s} X_{s}^{*}$ и $\varphi_{2}(s)=\widehat{B}_{s} \widehat{X}_{s}^{*}-B_{s} X_{s}^{*}$. Таким образом, из (7.2) получаем

$$
\begin{aligned}
\Delta_{t}^{2} & =\left(\int_{T_{0}}^{t} \varphi_{1}(s) \mathrm{d} s+\int_{T_{0}}^{t} \varphi_{2}(s) \mathrm{d} W_{s}\right)^{2} \\
& \leqslant 2\left(t-T_{0}\right) \int_{T_{0}}^{t} \varphi_{1}^{2}(s) \mathrm{d} s+2\left(\int_{T_{0}}^{t} \varphi_{2}(s) \mathrm{d} W_{s}\right)^{2} .
\end{aligned}
$$

Заметим, что

$$
\varphi_{1}^{2}(s) \leqslant\left(\left|\widehat{A}_{s}-A_{s}\right|\left|\widehat{X}_{s}^{*}\right|+\left|A_{s}\right|\left|\Delta_{s}\right|\right)^{2} \leqslant 2\left|\widehat{A}_{s}-A_{s}\right|^{2}\left|\widehat{X}_{s}^{*}\right|^{2}+2\left|A_{s}\right|^{2}\left|\Delta_{s}\right|^{2} .
$$

Так как $\widehat{B}_{s}=B_{s}$, то $\varphi_{2}^{2}(s) \leqslant\left(\left|\widehat{B}_{s}-B_{s}\right|\left|\widehat{X}_{s}^{*}\right|+\left|B_{s}\right|\left|\Delta_{s}\right|\right)^{2} \leqslant\left|B_{s}\right|^{2}\left|\Delta_{s}\right|^{2}$. Полагая $g(t)=\mathbf{E}_{T_{0}} \Delta_{t}^{2}$, получим

$$
g(t) \leqslant c_{0} \int_{T_{0}}^{t} g(s) \mathrm{d} s+\psi(t), \quad \text { где } \quad \psi(t)=4 \widetilde{T} \int_{T_{0}}^{t} \mathbf{E}_{T_{0}}\left(\left|\widehat{A}_{s}-A_{s}\right|^{2}\left|\widehat{X}_{s}^{*}\right|^{2}\right) \mathrm{d} s .
$$

Неравенство Гронуолла-Беллмана дает

$$
\begin{aligned}
g(t) & \leqslant \psi(t) e^{2 c_{0} t} \leqslant x^{2} \cdot 4 \widetilde{T} e^{c_{0} T} \int_{T_{0}}^{t} \mathbf{E}_{T_{0}}\left(\left|\widehat{A}_{s}-A_{s}\right|^{2}\left|\widehat{X}_{s}^{*}\right|^{2}\right) \mathrm{d} s \\
& \leqslant \widetilde{c}^{2} x^{2} \int_{T_{0}}^{t} \mathbf{E}_{T_{0}}\left|\widehat{A}_{s}-A_{s}\right|^{2} \mathrm{~d} s \leqslant \widetilde{c}^{2} x^{2} \int_{T_{0}}^{t} \mathbf{E}_{T_{0}}\left|\widehat{h}\left(s, Y_{s}\right)^{-q_{*}}-h\left(s, Y_{s}\right)^{-q_{*}}\right|^{2} \mathrm{~d} s \\
& \leqslant \widetilde{c}^{2} x^{2} q_{*} \int_{T_{0}}^{t} \mathbf{E}_{T_{0}}\left|\widehat{h}\left(s, Y_{s}\right)-h\left(s, Y_{s}\right)\right|^{2} \mathrm{~d} s .
\end{aligned}
$$

Воспользовавшись предложением 10.1 и леммой 10.6, получим, что для любого $T_{0} \leqslant s \leqslant T$

$$
\begin{aligned}
\mathbf{E}_{T_{0}}\left|\widehat{h}\left(s, Y_{s}\right)-h\left(s, Y_{s}\right)\right| & \leqslant \mathbf{h}_{1} \mathbf{E}_{T_{0}}\left(e^{\varkappa(T-s)}\left(\iota_{0}+\left|Y_{s}\right|\right)\right)|\bar{\alpha}| \\
& \leqslant \mathbf{h}_{1}\left(\iota_{0}+\mathbf{E}_{T_{0}}\left|Y_{s}\right|\right) e^{\varkappa(T-s)}|\bar{\alpha}| \\
& \leqslant \mathbf{h}_{1}\left(2 \iota_{0}+\left|Y_{T_{0}}\right|\right) e^{\varkappa(T-s)}|\bar{\alpha}| .
\end{aligned}
$$

Следовательно,

$$
g(t) \leqslant x^{2} \widetilde{c}^{2} q_{*}\left(\mathbf{h}_{1}\left(2 \iota_{0}+\left|Y_{T_{0}}\right|\right)\right)^{2} \frac{e^{2 \varkappa \widetilde{T}}}{\varkappa^{2}}|\bar{\alpha}|^{2},
$$


т.е. неравенство (8.15) выполняется. Докажем неравенство (8.16). Заметим, что в силу (7.1) оптимальная интенсивность потребления ограничена: $0 \leqslant c_{t}^{*} \leqslant 1$. Следовательно,

$$
\begin{aligned}
& \mathbf{E}_{T_{0}} \int_{T_{0}}^{T}\left|\widehat{c}_{t}^{*} \widehat{X}_{t}^{*}-c_{t}^{*} X_{t}^{*}\right|^{\gamma} \mathrm{d} t \\
& \quad \leqslant \mathbf{E}_{T_{0}} \int_{T_{0}}^{T}\left|\widehat{c}_{t}^{*}-c_{t}^{*}\right|^{\gamma}\left|\widehat{X}_{t}^{*}\right|^{\gamma} \mathrm{d} t+\int_{T_{0}}^{T} \mathbf{E}_{T_{0}}\left|\widehat{X}_{t}^{*}-X_{t}^{*}\right|^{\gamma} \mathrm{d} t \\
& \quad \leqslant x^{\gamma} \widetilde{d}^{\gamma} \mathbf{E}_{T_{0}} \int_{T_{0}}^{T}\left|\widehat{c}_{t}^{*}-c_{t}^{*}\right|^{\gamma} \mathrm{d} t+\widetilde{T} \sup _{T_{0} \leqslant t \leqslant T} \mathbf{E}_{T_{0}}\left|\widehat{X}_{t}^{*}-X_{t}^{*}\right|^{\gamma}
\end{aligned}
$$

Используя верхнюю границу (8.17) и принимая во внимание неравенство $\inf _{(t, y) \in \mathscr{K}} h(t, y) \geqslant 1$, получим

$$
\begin{aligned}
\mathbf{E}_{T_{0}} \int_{T_{0}}^{T}\left|\widehat{c}_{t}^{*}-c_{t}^{*}\right|^{\gamma} \mathrm{d} t & \leqslant q_{*} \int_{T_{0}}^{T} \mathbf{E}_{T_{0}}\left|\widehat{h}\left(s, Y_{s}\right)-h\left(s, Y_{s}\right)\right|^{\gamma} \mathrm{d} t \\
& \leqslant q_{*} \widetilde{T}\left(\mathbf{h}_{1}\left(2 \iota_{0}+\left|Y_{T_{0}}\right|\right)\right)^{\gamma} \frac{e^{\gamma \widetilde{T}}}{\varkappa^{\gamma}} \mathbf{E}_{T_{0}}|\bar{\alpha}|^{\gamma} .
\end{aligned}
$$

Значит,

$$
\mathbf{E}_{T_{0}} \int_{T_{0}}^{T}\left|\widehat{c}_{t}^{*} \widehat{X}_{t}^{*}-c_{t}^{*} X_{t}^{*}\right|^{\gamma} \mathrm{d} t \leqslant k_{2} x^{\gamma}\left(\mathbf{h}_{1}\left(2 \iota_{0}+\left|Y_{T_{0}}\right|\right)\right)^{\gamma} \mathbf{E}_{T_{0}}|\bar{\alpha}|^{\gamma} .
$$

Отсюда следует (8.16). Лемма 8.1 доказана.

8.3. Случай неизвестного $\mu$. На практике не реалистично рассматривать рынок с известным параметром $\mu$. В этом разделе мы обсудим модель, где неизвестным является не только параметр $\alpha$, но и параметр $\mu$. Напомним, что динамика акций задается уравнениями (2.1). Пусть оценка $\widehat{\mu}$ определена формулой

$$
\widehat{\mu}=\operatorname{Proj}_{\left[-\mu_{*}, \mu_{*}\right]}(\widetilde{\mu}), \quad \widetilde{\mu}=\frac{Z_{T_{0}}}{T_{0}}, \quad Z_{t}=\int_{0}^{t} \frac{1}{S_{t}} \mathrm{~d} S_{t} .
$$

Лемма 8.2. При любом $0<T_{0}<T$ справедливь следующие неравенства:

$$
\sup _{|\mu| \leqslant \mu_{*}} \mathbf{E}|\widehat{\mu}-\mu| \leqslant \epsilon_{1}\left(T_{0}\right) \quad u \quad \sup _{|\mu| \leqslant \mu_{*}} \mathbf{E}|\widehat{\mu}-\mu|^{2} \leqslant \epsilon_{1}^{2}\left(T_{0}\right)
$$

где $\epsilon_{1}\left(T_{0}\right)=\sigma^{*} / \sqrt{T_{0}}$ u $\sigma^{*}=\sup _{y \in \mathbf{R}} \sigma(y)$.

Д о к а з а т е л ь с т в о. Из определения процесса $Z$ следует, что

$$
\widetilde{\mu}-\mu=\frac{1}{T_{0}} \int_{0}^{T_{0}} \sigma\left(Y_{t}\right) \mathrm{d} W_{t}
$$


откуда непосредственно получаем оценки

$$
\sup _{|\mu| \leqslant \mu_{*}} \mathbf{E}|\widetilde{\mu}-\mu| \leqslant \epsilon_{1}\left(T_{0}\right) \quad \text { и } \quad \sup _{|\mu| \leqslant \mu_{*}} \mathbf{E}|\widetilde{\mu}-\mu|^{2} \leqslant \epsilon_{1}^{2}\left(T_{0}\right) .
$$

Учитывая, что $|\widehat{\mu}-\mu| \leqslant|\widetilde{\mu}-\mu|$, приходим к (8.19). Лемма 8.2 доказана. Пусть $J^{*}\left(T_{0}, x, y\right)$ — оптимальная целевая функция и ее оценка $\widehat{J}_{T_{0}}^{*}$ определена в (8.1). Введем константы

$$
k_{1}^{\prime}=2 \sqrt{\widetilde{c} \widetilde{T}} \frac{2 \mu_{*}+r+\sigma_{1}+1}{\sigma_{1}^{2}(1-\gamma)} \quad \text { и } \quad k_{2}^{\prime}=\frac{e^{\varkappa \widetilde{T}}}{\varkappa} .
$$

Кроме того, положим

$$
\begin{gathered}
k_{3}=\left(k_{1}^{\prime}\right)^{\gamma}+\left(\sqrt{2 \widetilde{c} q_{*}} k_{2}^{\prime} \mathbf{h}_{2}\right)^{\gamma}, \quad k_{4}=\left(\sqrt{2 \widetilde{c} q_{*}} k_{2}^{\prime} \mathbf{h}_{1}\right)^{\gamma}, \\
k_{5}=\widetilde{T}\left(k_{1}^{\prime}\right)^{\gamma}+k_{7}\left(k_{2}^{\prime} \mathbf{h}_{2}\right)^{\gamma}, \quad k_{6}=k_{7}\left(k_{2}^{\prime} \mathbf{h}_{1}\right)^{\gamma}, \quad k_{7}=\left(\sqrt{2 \widetilde{c} q_{*}}+q_{*} \widetilde{d^{\gamma}}\right),
\end{gathered}
$$

где параметры $\widetilde{c}, \mathbf{h}_{1}$ введены в (8.9) и

$$
\mathbf{h}_{2}=\frac{\gamma\left(\mu_{*}+r\right)}{(1-\gamma) \sigma_{1}^{2}} \frac{2 \widetilde{T}^{2}}{\iota_{0}}
$$

Теорема 8.2. Оченка $\widehat{J}_{T_{0}}^{*}$ оптимальной челевой функции удовлетворяет следуюшему неравенству:

$$
\left|\widehat{J}_{T_{0}}^{*}-J^{*}\left(T_{0}, x, Y_{T_{0}}\right)\right| \leqslant x^{\gamma} \Gamma_{1}\left(2 \iota_{0}+\left|Y_{T_{0}}\right|\right)^{\gamma} \varpi^{\gamma}+x^{\gamma} \Gamma_{2}\left(2 \iota_{0}+\left|Y_{T_{0}}\right|\right)^{\gamma}|\bar{\alpha}|^{\gamma},
$$

где $\Gamma_{1}=k_{3}+k_{5}, \Gamma_{2}=k_{4}+k_{6} u \varpi=|\widehat{\mu}-\mu|+|\widehat{\mu}-\mu|^{2}$. Кроме того, для любьх $m \geqslant 1$

$$
\sup _{\alpha_{2} \leqslant \alpha \leqslant \alpha_{1}} \sup _{|\mu| \leqslant \mu_{*}} \mathbf{E}\left|\widehat{J}_{T_{0}}^{*}-J^{*}\left(T_{0}, x, Y_{T_{0}}\right)\right| \leqslant \check{\delta}_{m},
$$

где $\check{\delta}_{m}=\check{\delta}_{m}\left(x, T_{0}\right)=x^{\gamma}\left(\Gamma_{1}\left(3 \iota_{0}^{\gamma}+\left|Y_{0}\right|^{\gamma}\right) \epsilon_{2}^{\gamma}\left(T_{0}\right)+\check{\Gamma}_{m} \epsilon^{\gamma}\left(T_{0}\right)\right) u$

$$
\check{\Gamma}_{m}=\Gamma_{2}\left(\left(2 \iota_{0}\right)^{\gamma}+\left(\frac{(2 m-1) ! ! \beta^{2 m}}{\left(2\left|\alpha_{1}\right|\right)^{m}}\right)^{\gamma / 2 m}\right) \text {. }
$$

Здесь $\iota_{0}=\beta / \sqrt{2\left|\alpha_{1}\right|}, \epsilon_{2}\left(T_{0}\right)=\epsilon_{1}\left(T_{0}\right)+\epsilon_{1}^{2}\left(T_{0}\right)$, функиии $\epsilon_{1}(\cdot)$ и $\epsilon(\cdot)$ определень в (8.19) и (8.7) соответственно.

Д о к а з а т е л ь с т в о. Чтобы получить (8.21), мы используем то же рассуждение, что в теореме 8.1, и лемму 8.3. Для доказательства (8.22) заметим, что

$$
\begin{aligned}
\mathbf{E}\left|\widehat{J}_{T_{0}}^{*}-J^{*}\left(T_{0}, x, Y_{T_{0}}\right)\right| \leqslant & x^{\gamma} \Gamma_{1}\left(\left(2 \iota_{0}\right)^{\gamma}+\left(\mathbf{E}\left|Y_{T_{0}}\right|\right)^{\gamma}\right) \mathbf{E} \varpi^{\gamma} \\
& +x^{\gamma} \Gamma_{2}\left(2 \iota_{0}\right)^{\gamma} \epsilon^{\gamma}\left(T_{0}\right)+\mathbf{E}\left(\left|Y_{T_{0}}\right|^{\gamma}|\bar{\alpha}|^{\gamma}\right) .
\end{aligned}
$$

Учитывая, что $\mathbf{E} \varpi \leqslant \epsilon_{2}\left(T_{0}\right)$, и используя неравенство (8.13), мы получаем (8.22). Теорема 8.2 доказана. 
Лемма 8.3. Оцениваемьй процесс капитала $\left(\widehat{X}_{t}^{*}\right)_{T_{0} \leqslant t \leqslant T}$ удовлетворяет следуюшим неравенствам:

$$
\begin{aligned}
\sup _{T_{0} \leqslant t \leqslant T} \mathbf{E}_{T_{0}}\left|\widehat{X}_{t}^{*}-X_{t}^{*}\right|^{\gamma} \leqslant & x^{\gamma} k_{3}\left(2 \iota_{0}+\left|Y_{T_{0}}\right|\right)^{\gamma} \varpi^{\gamma} \\
& +x^{\gamma} k_{4}\left(2 \iota_{0}+\left|Y_{T_{0}}\right|\right)^{\gamma}|\bar{\alpha}|^{\gamma}, \\
\mathbf{E}_{T_{0}} \int_{T_{0}}^{T}\left|\widehat{c}_{t}^{*} \widehat{X}_{t}^{*}-c_{t}^{*} X_{t}^{*}\right|^{\gamma} \mathrm{d} t \leqslant & x^{\gamma} k_{5}\left(2 \iota_{0}+\left|Y_{T_{0}}\right|\right)^{\gamma} \varpi^{\gamma} \\
& +x^{\gamma} k_{6}\left(2 \iota_{0}+\left|Y_{T_{0}}\right|\right)^{\gamma}|\bar{\alpha}|^{\gamma} .
\end{aligned}
$$

Д о к а з а т е л ь с т в о. Следуя доказательству леммы 8.1, положим $\Delta_{t}=\widehat{X}_{t}^{*}-X_{t}^{*}, g(t)=\mathbf{E}_{T_{0}} \Delta_{t}^{2}$. Тогда

$$
g(t) \leqslant c_{0} \int_{T_{0}}^{t} g(s) \mathrm{d} s+\psi(t),
$$

где $\psi(t)=4 \mathbf{E}_{T_{0}} \int_{T_{0}}^{t}\left(\left|\widehat{A}_{s}-A_{s}\right|^{2}+\left|\widehat{B}_{s}-B_{s}\right|^{2}\right)\left|\widehat{X}_{s}^{*}\right|^{2} \mathrm{~d} s$. Воспользовавшись неравенством Гронуолла-Беллмана, приходим к следующей оценке:

$$
\begin{aligned}
& g(t) \leqslant \psi(t) e^{c_{0} t} \leqslant x^{2} \widetilde{c} \int_{T_{0}}^{t} \mathbf{E}_{T_{0}}\left(\left|\widehat{A}_{s}-A_{s}\right|^{2}+\left|\widehat{B}_{s}-B_{s}\right|^{2}\right) \mathrm{d} s \\
& \leqslant x^{2} \widetilde{c} \widetilde{T} \frac{2\left(2 \mu_{*}+r\right)^{2}+\sigma_{1}^{2}}{\sigma_{1}^{4}(1-\gamma)^{2}} \varpi^{2}+2 x^{2} \widetilde{c} \int_{T_{0}}^{t} \mathbf{E}_{T_{0}}\left|\widehat{h}\left(s, Y_{s}\right)^{-q_{*}}-h\left(s, Y_{s}\right)^{-q_{*}}\right|^{2} \mathrm{~d} s \\
& \quad \leqslant 2 x^{2} \widetilde{c} \widetilde{T}\left(\frac{2 \mu_{2}+r+\sigma_{1}}{\sigma_{1}^{2}(1-\gamma)}\right)^{2} \varpi^{2}+2 x^{2} \widetilde{c} q_{*} \int_{T_{0}}^{t} \mathbf{E}_{T_{0}}\left|\widehat{h}\left(s, Y_{s}\right)-h\left(s, Y_{s}\right)\right|^{2} \mathrm{~d} s .
\end{aligned}
$$

Мы используем предложение 10.2, чтобы получить аналог неравенства (8.17):

$$
\mathbf{E}_{T_{0}}\left|\widehat{h}\left(s, Y_{s}\right)-h\left(s, Y_{s}\right)\right| \leqslant e^{\varkappa(T-s)}\left(2 \iota_{0}+\left|Y_{T_{0}}\right|\right) \tilde{\delta}
$$

где $\widetilde{\delta}=\mathbf{h}_{2} \varpi+\mathbf{h}_{1}|\bar{\alpha}|$. Далее,

$$
\begin{aligned}
g(t) & \leqslant 2 x^{2} \widetilde{c} \widetilde{T}\left(\frac{2 \mu_{*}+r+\sigma_{1}}{\sigma_{1}^{2}(1-\gamma)}\right)^{2} \varpi^{2}+2 x^{2} \widetilde{c} q_{*} \frac{e^{2 \varkappa \widetilde{T}}}{\varkappa^{2}}\left(2 \iota_{0}+\left|Y_{T_{0}}\right|\right)^{2} \widetilde{\delta}^{2} \\
& \leqslant x^{2}\left(k_{1}^{\prime} \varpi+k_{2}^{\prime}\left(2 \iota_{0}+\left|Y_{T_{0}}\right|\right) \widetilde{\delta}\right)^{2}
\end{aligned}
$$

так что

$$
\mathbf{E}_{T_{0}}\left|\Delta_{t}\right| \leqslant x\left(k_{1}^{\prime} \varpi+k_{2}^{\prime}\left(2 \iota_{0}+\left|Y_{T_{0}}\right|\right) \widetilde{\delta}\right) .
$$

Используя неравенство Йенсена для степенной функции $z^{\gamma}($ с $0<\gamma<1)$, получаем (8.23). Теперь докажем (8.24). Мы рассуждаем так же, как при доказательстве лемме 8.1. Имеем

$$
\begin{aligned}
\mathbf{E} \int_{T_{0}}^{T}\left|\widehat{c}_{t}^{*} \widehat{X}_{t}^{*}-c_{t}^{*} X_{t}^{*}\right|^{\gamma} \mathrm{d} t \leqslant & x^{\gamma} q_{*} \tilde{d}^{\gamma} \int_{T_{0}}^{T} \mathbf{E}\left|\widehat{h}\left(s, Y_{s}\right)-h\left(s, Y_{s}\right)\right|^{\gamma} \mathrm{d} s \\
& +\widetilde{T} \sup _{T_{0} \leqslant t \leqslant T} \mathbf{E}\left|\widehat{X}_{t}^{*}-X_{t}^{*}\right|^{\gamma} .
\end{aligned}
$$


Следовательно, верхняя граница (8.24) немедленно вытекает из (8.23) и (8.25). Лемма 8.3 доказана.

9. Моделирование. В этом разделе мы используем для моделирования пакет программ Scilab. На рис. 1 мы моделируем усеченную последовательную процедуру $\widehat{\alpha}$ для различных значений $T_{0}$ по 30 траекториям процесса $Y$. Последовательные оценки представлены крестиками $\times$ для $T_{0}$, равного 5 дням, и звездочками $\star$ для $T_{0}$, равного 10 дням.

Истинное значение сноса процесса $Y$ равно $\alpha=-5$. Мы берем границы $\alpha \in[-0.15,-10]$ и полагаем $\beta=1$.

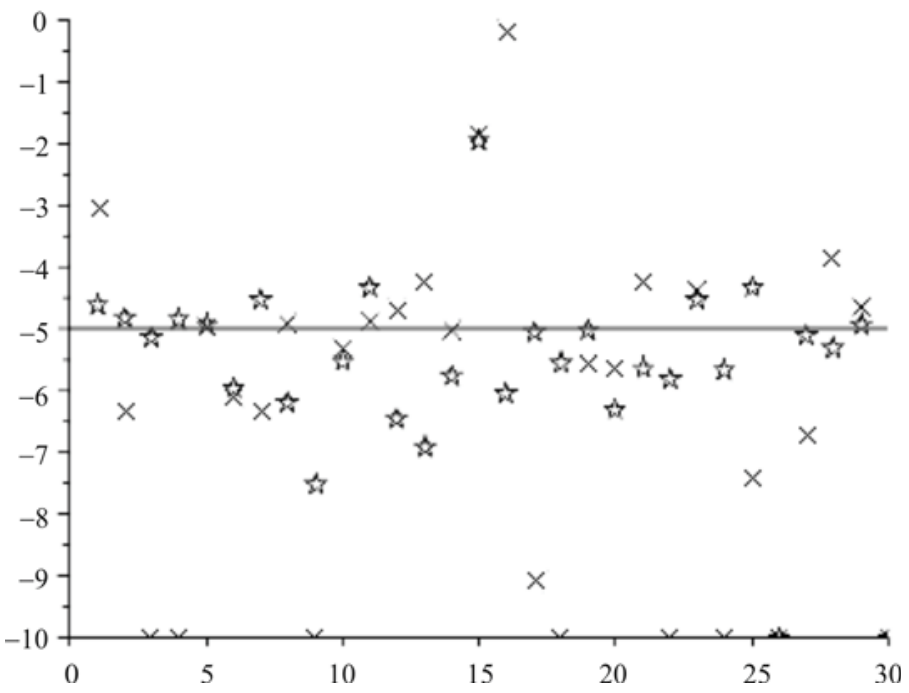

Рис. 1. Усеченная последовательная процедура для $T_{0}=5$ и $T_{0}=10$.

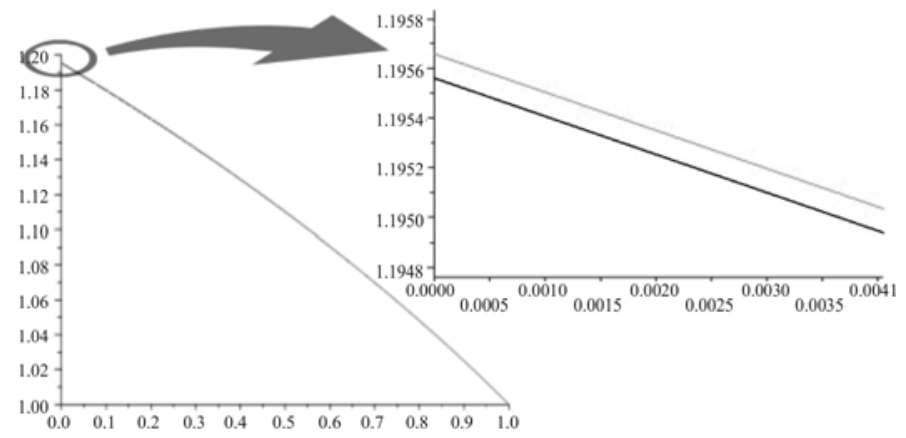

Рис. 2. Предельные функции $h(t, 0)$ и $\widehat{h}(t, 0)$.

На рис. 2 мы моделируем предельные функции $h(t, y)$ и $\widehat{h}(t, y)$ при следующих настройках рынка: $T_{0}=5$ и $\widetilde{T}=T-T_{0}=1, r=0.01$, $\mu=0.02$. Параметр волатильности определен как $\sigma(y)=0.5+\sin ^{2} y$. Параметр полезности $\gamma$ равен 0.75. Для моделирования $\widehat{h}(t, y)$ мы используем очень пессимистическую реализацию усеченной оценки, а именно 
$\widehat{\alpha}=-0.5$. Истинное значение $\alpha$ равно -5 . Мы видим, что даже в этой крайней ситуации функция $\widehat{h}(t, y)$ не очень существенно отклоняется от истинной функции $h(t, y)$.

10. Приложение. Пусть $h$ - неподвижная точка для $\mathscr{L}$, т.е. $h=$ $\mathscr{L}_{h}$. Напомним, что оператор $\mathscr{L}$ определен в $(4.4),(4.5)$. В этом разделе мы изучаем свойства непрерывности по параметрам $\alpha$ и $\mu$ функции $h$. Для этого мы исследуем сначала частные производные функций $\mathscr{H}_{f}(t, s, y)$ и $h$ по $y$.

Лемма 10.1. Для любьх $t$ u $s$ mаких, ито $T_{0}<t \leqslant s \leqslant T$,

$$
\sup _{y \in \mathbf{R}} \sup _{f \in \mathscr{X}}\left|\frac{\partial}{\partial y} \mathscr{H}_{f}(t, s, y)\right| \leqslant Q_{1}^{*} \widetilde{T} e^{Q_{*} \widetilde{T}}+\frac{e^{Q_{*} \widetilde{T}}}{\nu_{s}}
$$

где $\nu_{s}^{2}=\beta^{2}\left(1-e^{2 \alpha(s-t)}\right) /(2|\alpha|)$.

Д о к а з а т е л ь с т в о. Чтобы вычислить условное математическое ожидание в $(4.5)$, заметим сначала, что

$$
\eta_{s}=y e^{\alpha(s-t)}+\int_{t}^{s} \beta e^{\alpha(s-v)} \mathrm{d} \widetilde{\mathbf{U}}_{v}=y e^{\alpha(s-t)}+\xi_{s} .
$$

Так как $\eta$ является гауссовским прочессом, то для любых $t<v_{1}<\cdots<$ $v_{k}<s$ и любых ограниченных функций $G: \mathbf{R}^{k} \rightarrow \mathbf{R}$ выполнено равенство

$$
\mathbf{E}\left(G\left(\eta_{v_{1}}, \ldots, \eta_{v_{k}}\right) \mid \eta_{s}=z\right)=\mathbf{E} G\left(\mathbf{B}_{v_{1}}, \ldots, \mathbf{B}_{v_{k}}\right)
$$

где $\mathbf{B}_{v}=\eta_{v}-k(v) \eta_{s}+k(v) z$ и коэффициент $k(v)$ выбран так, что

$$
\mathbf{E}\left[\left(\xi_{v}-k(v) \xi_{s}\right) \xi_{s}\right]=0, \quad \text { т.е. } \quad k(v)=\frac{\mathbf{E}\left(\xi_{v} \xi_{s}\right)}{\mathbf{E} \xi_{s}^{2}}=e^{\alpha(s-v)} \frac{1-e^{2 \alpha(v-t)}}{1-e^{2 \alpha(s-t)}} \leqslant 1
$$

Условное математическое ожидание относительно $\eta_{s}$ позволяет представить $\mathscr{H}_{f}$ как

$$
\begin{aligned}
\mathscr{H}_{f}(t, s, y) & =\int_{\mathbf{R}} \widehat{\mathscr{H}}_{f}(s, y, z) \mathbf{p}(z, y) \mathrm{d} z, \\
\mathbf{p}(z, y) & =\frac{1}{\nu_{s} \sqrt{2 \pi}} \exp \left\{-\frac{(z-\mu(y))^{2}}{2 \nu_{s}^{2}}\right\},
\end{aligned}
$$

где $\mu(y)=\mathbf{E} \eta_{s}=y e^{\alpha(s-t)}$ и $\nu_{s}^{2}=\mathbf{D} \eta_{s}$. Так как $\mathbf{B}_{s}=z$, мы получаем

$$
\begin{aligned}
\widehat{\mathscr{H}}_{f}(s, y, z) & =\mathbf{E}\left(\left(f\left(s, \eta_{s}^{t, y}\right)\right)^{1-q_{*}} \exp \left\{\int_{t}^{s} Q\left(\eta_{u}^{t, y}\right) \mathrm{d} u\right\} \mid \eta_{s}=z\right) \\
& =\mathbf{E}\left((f(s, z))^{1-q_{*}} \exp \left\{\int_{t}^{s} Q\left(\mathbf{B}_{u}\right) \mathrm{d} u\right\}\right) \leqslant e^{Q_{*}(s-t)} .
\end{aligned}
$$


Отсюда следует, что

$\left|\frac{\partial}{\partial y} \widehat{\mathscr{H}}_{f}(s, y, z)\right| \leqslant\left|\int_{t}^{s} \frac{\partial Q\left(\mathbf{B}_{u}\right)}{\partial y} \mathrm{~d} u\right| \widehat{\mathscr{H}}_{f}(s, y, z) \leqslant Q_{1}^{*}(s-t) e^{Q_{*}(s-t)} \leqslant Q_{1}^{*} \widetilde{T} e^{Q_{*} \widetilde{T}}$.

Теперь из (10.3) получаем

$$
\begin{aligned}
\frac{\partial \mathscr{H}_{f}(t, s, y)}{\partial y}= & \int_{\mathbf{R}} \frac{\partial \widehat{\mathscr{H}}_{f}(s, y, z)}{\partial y} \mathbf{p}(z, y) \mathrm{d} z \\
& +\int_{\mathbf{R}} \widehat{\mathscr{H}}_{f}(s, y, z) \frac{(z-\mu(y)) \mu^{\prime}(y)}{\nu_{s}^{2}} \mathbf{p}(z, y) \mathrm{d} z
\end{aligned}
$$

Следовательно,

$$
\begin{aligned}
\left|\frac{\partial \mathscr{H}_{f}(t, s, y)}{\partial y}\right| & \leqslant Q_{1}^{*}(s-t) e^{Q_{*}(s-t)}+e^{Q_{*}(s-t)} \frac{\mu^{\prime}(y)}{\nu_{s}^{2}} \int_{\mathbf{R}}|z-\mu(y)| \mathbf{p}(z, y) \mathrm{d} z \\
& \leqslant Q_{1}^{*}(s-t) e^{Q_{*}(s-t)}+\frac{e^{\left(Q_{*}+\alpha\right)(s-t)}}{\nu_{s}^{2}} \frac{2 \nu_{s}}{\sqrt{2 \pi}} \leqslant Q_{1}^{*} \widetilde{T} e^{Q_{*} \widetilde{T}}+\frac{e^{Q_{*} \widetilde{T}}}{\nu_{s}} .
\end{aligned}
$$

Таким образом, лемма 10.1 доказана.

Лемма 10.2. Неподвижная точка $h=\mathscr{L}_{h}$ дифферениируема по $y \in \mathbf{R}$, и ее частная производная ограничена:

$$
\sup _{T_{0} \leqslant t \leqslant T, y \in \mathbf{R}}\left|\frac{\partial}{\partial y} h(t, y)\right| \leqslant h_{1}^{*},
$$

где $h_{1}^{*}$ определено в (8.10).

Д о к а з а т е л ь с т в о. Ясно, что достаточно показать, что функция $\mathscr{L}_{f}(t, y)$ дифференцируема по $y$ и ее частная производная ограничена:

$$
\sup _{T_{0} \leqslant t \leqslant T, y \in \mathbf{R}}\left|\frac{\partial}{\partial y} \mathscr{L}_{f}(t, y)\right| \leqslant h_{1}^{*}
$$

Из определения $\mathscr{L}_{f}$ (см. (4.4)) для всех $f \in \mathscr{X}$ и всех $t \in\left[T_{0}, T\right], y \in \mathbf{R}$ следует, что

$$
\frac{\partial}{\partial y} \mathscr{L}_{f}(t, y)=\mathbf{E} \frac{\partial}{\partial y} \mathscr{G}(t, T, y)+\frac{1}{q_{*}} \int_{t}^{T} \frac{\partial}{\partial y} \mathscr{H}_{f}(t, s, y) \mathrm{d} s .
$$

С помощью леммы 10.1 и приводимой далее леммы 10.4 получаем

$$
\begin{aligned}
\sup _{T_{0} \leqslant t \leqslant T, y \in \mathbf{R}}\left|\frac{\partial}{\partial y} \mathscr{L}_{f}(t, y)\right| & \leqslant \widetilde{T} Q_{1}^{*} e^{Q_{*} \widetilde{T}}+\frac{1}{q_{*}} \int_{t}^{T} Q_{1}^{*} \widetilde{T} e^{Q_{*} \widetilde{T}} \mathrm{~d} s+\frac{1}{q_{*}} \int_{t}^{T} \frac{e^{Q_{*} \widetilde{T}}}{\nu_{s}} \mathrm{~d} s \\
& \leqslant \widetilde{T} Q_{1}^{*} e^{Q_{*} \widetilde{T}}+\frac{Q_{1}^{*} \widetilde{T}^{2}}{q_{*}} e^{Q_{*} \widetilde{T}}+\frac{e^{Q_{*} \widetilde{T}}}{q_{*}} \int_{t}^{T} \frac{1}{\nu_{s}} \mathrm{~d} s .
\end{aligned}
$$


Чтобы оценить $\int_{t}^{T}\left(1 / \nu_{s}\right) \mathrm{d} s$, заметим, что $2|\alpha|(s-t) \leqslant 2|\alpha| \widetilde{T}$ и, значит,

$$
\begin{aligned}
& \nu_{s}^{2}=\beta^{2} \frac{1-e^{2 \alpha(s-t)}}{2|\alpha|(s-t)}(s-t) \geqslant \beta^{2} \frac{1-e^{2 \alpha}}{2|\alpha|}(s-t), \quad \text { если } s-t \leqslant 1, \\
& \nu_{s}^{2}=\beta^{2} \frac{1-e^{2 \alpha(s-t)}}{2|\alpha|} \geqslant \beta^{2} \frac{1-e^{2 \alpha}}{2|\alpha|}, \quad \text { если } s-t \geqslant 1 .
\end{aligned}
$$

Следовательно,

$$
\begin{aligned}
\int_{t}^{T} \frac{1}{\nu_{s}} \mathrm{~d} s & \leqslant \sqrt{\frac{2|\alpha|}{\beta^{2}\left(1-e^{2 \alpha}\right)}} \int_{t}^{t+1} \frac{1}{\sqrt{s-t}} \mathrm{~d} s+\sqrt{\frac{2|\alpha|}{\beta^{2}\left(1-e^{2 \alpha}\right)}} \int_{t+1}^{T} \mathrm{~d} s \\
& \leqslant 2 \sqrt{\frac{2|\alpha|}{\beta^{2}\left(1-e^{2 \alpha}\right)}}+\sqrt{\frac{2|\alpha|}{\beta^{2}\left(1-e^{2 \alpha}\right)}} \widetilde{T} \leqslant 3 \sqrt{\frac{2|\alpha|}{\beta^{2}\left(1-e^{2 \alpha}\right)}} \widetilde{T} .
\end{aligned}
$$

Учитывая, что $\alpha_{2} \leqslant \alpha \leqslant \alpha_{1}<0$, мы получаем желаемый результат. Лемма 10.2 доказана.

Сейчас мы изучим частные производные функции $\mathscr{G}(t, s, y)$, определенной в (4.4). Для этого нам нужен следующий результат.

Лемма 10.3. Пусть $F=F(y, \omega): \mathbf{R} \times \Omega \rightarrow \mathbf{R}-$ случайная ограниченная функция такая, что для некоторой неслучайной константы $c^{*}$

$$
\left|\frac{\mathrm{d}}{\mathrm{d} y} F(y, \omega)\right| \leqslant c^{*} \quad \text { n.н. }
$$

Тогда

$$
\frac{\mathrm{d}}{\mathrm{d} y} \mathbf{E} F(y, \omega)=\mathbf{E} \frac{\mathrm{d}}{\mathrm{d} y} F(y, \omega) .
$$

Эта лемма непосредственно вытекает из теоремы Лебега о мажорируемой сходимости.

Лемма 10.4. Для любых $t<s$ функиия $\mathscr{G}$ удовлетворяет следующим соотношениям:

$$
\sup _{y \in \mathbf{R}}\left|\frac{\partial \mathscr{G}(t, s, y)}{\partial y}\right| \leqslant(s-t) Q_{1}^{*} e^{Q_{*}(s-t)}, \quad \frac{\partial}{\partial y} \mathbf{E} \mathscr{G}(t, s, y)=\mathbf{E} \frac{\partial}{\partial y} \mathscr{G}(t, s, y) .
$$

Д о к а з а те ль с т в о. Имеем

$$
\frac{\partial \mathscr{G}(t, s, y)}{\partial y}=\mathscr{G}(t, s, y) \mathbf{G}(t, s, y)
$$

где $\mathbf{G}(t, s, y)=\int_{t}^{s} Q_{1}\left(\eta_{u}^{t, y}\right)\left(\partial \eta_{u}^{t, y} / \partial y\right) \mathrm{d} u$ и $Q_{1}(z)=\mathrm{d} Q(z) / \mathrm{d} z$. Теперь лемма 10.4 следует непосредственно из леммы 10.3 .

Лемма 10.5. Для любой функиии $f \in \mathscr{X}$, имеющей ограниченную частную производную по $y \in \mathbf{R}, u$ для любого $N>0$

$$
\sup _{|y|<N} \sup _{0 \leqslant t_{1}<t_{2} \leqslant T} \frac{\left|\mathscr{L}_{f}\left(t_{2}, y\right)-\mathscr{L}_{f}\left(t_{1}, y\right)\right|}{\sqrt{t_{2}-t_{1}}}<\infty .
$$


Д о к а з а т е л ь с т в о. Можно проверить, что

$$
\sup _{|y|<N} \sup _{0 \leqslant t_{1}<t_{2} \leqslant s \leqslant T} \frac{\mathbf{E}\left|\eta_{s}^{t_{2}, y}-\eta_{s}^{t_{1}, y}\right|}{\sqrt{t_{2}-t_{1}}}<\infty .
$$

Отсюда получаем лемму 10.5 .

Напомним, что процессы $\left(\eta_{s}\right)_{0 \leqslant s \leqslant T}$ и $\left(\widehat{\eta}_{s}^{t, y}\right)_{0 \leqslant s \leqslant T}$ определены в $(4.3)$ и (8.5) соответственно.

Лемма 10.6. Для любых $T_{0} \leqslant t \leqslant s \leqslant T$

$$
\begin{gathered}
\mathbf{E}_{T_{0}}\left|\widehat{\eta}_{s}^{t, y}\right| \leqslant \iota_{0}+|y|=\frac{\beta}{\sqrt{2\left|\alpha_{1}\right|}}+|y|:=\widetilde{m}(y), \\
\mathbf{E}_{T_{0}} \int_{t}^{T}\left|\widehat{\eta}_{s}^{t, y}-\eta_{s}^{t, y}\right| \mathrm{d} t \leqslant \frac{\widetilde{T} \widetilde{m}(y)}{\left|\alpha_{1}\right|}|\bar{\alpha}| .
\end{gathered}
$$

Кроме того, для известного параметра $\mu$ и неизвестного $\alpha$

$$
\mathbf{E}_{T_{0}}|\widehat{\mathscr{G}}(t, s, y)-\mathscr{G}(t, s, y)| \leqslant \widetilde{T} Q_{1}^{*} e^{Q_{*}(T-t)} \frac{\widetilde{m}(y)}{\left|\alpha_{1}\right|}|\bar{\alpha}|,
$$

где $Q^{*}$ и $Q_{1}^{*}$ определеньв в (3.5), а функиия $\widehat{\mathscr{G}}(t, s, y)$ определена в (8.4).

Д о к а з а т е ль с т в о. Так как $\eta_{s}=\eta_{t} e^{\alpha(s-t)}+\int_{t}^{s} \beta e^{\alpha(s-v)} \mathrm{d} \widetilde{\mathbf{U}}_{v}$, то для любого $\alpha_{2} \leqslant \alpha \leqslant \alpha_{1}<0$

$$
\begin{aligned}
\mathbf{E}\left(\eta_{s}^{t, y}\right)^{2} & =y^{2} e^{2 \alpha(s-t)}+\beta^{2} \int_{t}^{s} e^{2 \alpha(t-v)} \mathrm{d} v \leqslant y^{2}+\frac{\beta^{2}}{2\left|\alpha_{1}\right|} \\
& \leqslant\left(|y|+\frac{\beta}{\sqrt{2\left|\alpha_{1}\right|}}\right)^{2} .
\end{aligned}
$$

Отсюда следует (10.7). Более того, полагая $\bar{\eta}_{s}=\widehat{\eta}_{s}^{t, y}-\eta_{s}^{t, y}$, получаем

$$
\mathrm{d} \bar{\eta}_{s}=\left(\widehat{\alpha} \widehat{\eta}_{s}^{t, y}-\alpha \eta_{s}^{t, y}\right) \mathrm{d} s=\alpha \bar{\eta}_{s} \mathrm{~d} s+\bar{\alpha} \widehat{\eta}_{s}^{t, y} \mathrm{~d} s,
$$

т.е. $\bar{\eta}_{s}=\int_{t}^{s} \bar{\alpha} e^{\alpha(s-u)} \hat{\eta}_{u}^{t, y} \mathrm{~d} u$. Следовательно, $\left|\bar{\eta}_{s}\right| \leqslant|\bar{\alpha}| \int_{t}^{s}\left|\widehat{\eta}_{u}^{t, y}\right| e^{\alpha(s-u)} \mathrm{d} u$. Так как $\widehat{\alpha}$ независима от броуновского движения $\left(\widetilde{\mathbf{U}}_{t}\right)$, мы получаем

$$
\begin{aligned}
\mathbf{E}_{T_{0}}\left|\bar{\eta}_{s}\right| & \leqslant|\bar{\alpha}| \mathbf{E}_{T_{0}} \int_{t}^{s}\left|\widehat{\eta}_{u}^{t, y}\right| e^{\alpha(s-u)} \mathrm{d} u \\
& \leqslant|\bar{\alpha}| \int_{t}^{s} e^{\alpha(s-u)} \mathbf{E}_{T_{0}}\left|\widehat{\eta}_{u}^{t, y}\right| \mathrm{d} u \leqslant \frac{\widetilde{m}(y)}{\left|\alpha_{1}\right|}|\bar{\alpha}| .
\end{aligned}
$$

Следовательно, для всех $T_{0} \leqslant t \leqslant T$

$$
\begin{aligned}
\mathbf{E}_{T_{0}} \int_{t}^{T}\left|\bar{\eta}_{s}\right| \mathrm{d} s & \leqslant \mathbf{E}_{T_{0}}\left(\int_{t}^{T}|\bar{\alpha}| \int_{t}^{s} e^{\alpha(s-u)}\left|\widehat{\eta}_{u}^{t, y}\right| \mathrm{d} u \mathrm{~d} s\right) \\
& \leqslant \widetilde{T}|\bar{\alpha}| \int_{t}^{T} e^{\alpha(s-u)} \mathbf{E}_{T_{0}}\left|\widehat{\eta}_{u}^{t, y}\right| \mathrm{d} u \leqslant \frac{\widetilde{m}(y) \widetilde{T}}{\left|\alpha_{1}\right|}|\bar{\alpha}|,
\end{aligned}
$$


и мы получаем (10.8). Чтобы доказать неравенство (10.9), заметим, что

$$
\begin{aligned}
|\widehat{\mathscr{G}}(t, s, y)-\mathscr{G}(t, s, y)| & \leqslant e^{Q_{*}(T-t)}\left|\int_{t}^{s} Q\left(\widehat{\eta}_{u}^{t, y}\right) \mathrm{d} u-\int_{t}^{s} Q\left(\eta_{u}^{t, y}\right) \mathrm{d} u\right| \\
& \leqslant e^{Q_{*}(T-t)} \int_{t}^{s} \sup _{y \in \mathbf{R}}\left|\frac{\partial Q(y)}{\partial y}\right|\left|\bar{\eta}_{u}\right| \mathrm{d} u \\
& \leqslant Q_{1}^{*} e^{Q_{*}(T-t)} \int_{t}^{T}\left|\bar{\eta}_{u}\right| \mathrm{d} u
\end{aligned}
$$

Поэтому

$$
\mathbf{E}_{T_{0}}|\widehat{\mathscr{G}}(t, s, y)-\mathscr{G}(t, s, y)| \leqslant Q_{1}^{*} e^{Q_{*}(T-t)} \int_{t}^{T} \mathbf{E}_{T_{0}}\left|\bar{\eta}_{u}\right| \mathrm{d} u .
$$

Отсюда и из (10.10) следует (10.9). Таким образом, лемма 10.6 доказана.

В следующем предложении мы изучим поведение функции $h(t, y)$, являющейся решением уравнения $h=\mathscr{L}_{h}$, используя оценку $\widehat{\alpha}$ для параметра $\alpha$. Нам надо изучить отклонение

$$
\bar{h}(t, y)=\widehat{h}(t, y)-h(t, y)
$$

где $\widehat{h}=\widehat{\mathscr{L}}_{\hat{h}}$. Оператор $\widehat{\mathscr{L}}$ определен в (8.4). Аналогично (4.2) мы определим на $\mathscr{X}$ метрику $\widetilde{\varrho}_{*}$ :

$$
\widetilde{\varrho}_{*}(f, g)=\sup _{(t, y) \in \mathscr{K}} e^{-\varkappa(T-t)} \frac{|f(t, y)-g(t, y)|}{\iota_{0}+|y|},
$$

где $\iota_{0}=\beta / \sqrt{2 \alpha_{1}}, \varkappa=Q_{*}+\zeta+1$ и $\zeta=\zeta_{0}+2 \gamma$ для некоторого $\zeta_{0}>0$.

Предложение 10.1. Для известного $\mu$ и неизвестного $\alpha$ и для любого $0<T_{0}<T$

$$
\widetilde{\varrho}_{*}(\widehat{h}, h) \leqslant \mathbf{h}_{1}|\bar{\alpha}|,
$$

где константа $\mathbf{h}_{1}$ определена в (8.9).

Д о к а з а т е л ь с т в о. Мы используем определение (4.4) оператора $\mathscr{L}$ :

$$
h(t, y)=\mathscr{L}_{h}(t, y)=\mathbf{E} \mathscr{G}(t, T, y)+\frac{1}{q_{*}} \int_{t}^{T} \mathscr{H}_{h}(t, s, y) \mathrm{d} s .
$$

С помощью (4.5) мы можем оценить отклонение (10.11) следующим обра30M:

$$
|\bar{h}(t, y)| \leqslant \mathbf{E}_{T_{0}}|\widehat{\mathscr{G}}(t, T, y)-\mathscr{G}(t, T, y)|+I(\widehat{\alpha}),
$$

где

$$
I(\widehat{\alpha})=\frac{1}{q_{*}} \int_{t}^{T} \mathbf{E}_{T_{0}}\left|\left(\widehat{h}\left(s, \widehat{\eta}_{s}^{t, y}\right)\right)^{1-q_{*}} \widehat{\mathscr{G}}(t, s, y)-\left(h\left(s, \eta_{s}^{t, y}\right)\right)^{1-q_{*}} \mathscr{G}(t, s, y)\right| \mathrm{d} s .
$$


Более того, эта функция допускает следующую оценку:

$$
\begin{aligned}
I(\widehat{\alpha}) \leqslant & \frac{1}{q_{*}} \int_{t}^{T} \mathbf{E}_{T_{0}}\left(h\left(s, \eta_{s}^{t, y}\right)^{1-q_{*}}|\widehat{\mathscr{G}}(t, s, y)-\mathscr{G}(t, s, y)|\right) \mathrm{d} s \\
& +\frac{1}{q_{*}} \int_{t}^{T} \mathbf{E}_{T_{0}}\left|\left(\widehat{h}\left(s, \widehat{\eta}_{s}^{t, y}\right)\right)^{1-q_{*}}-\left(h\left(s, \eta_{s}^{t, y}\right)\right)^{1-q_{*}}\right| e^{Q_{*}(s-t)} \mathrm{d} s \\
\leqslant & \int_{t}^{T} \mathbf{E}_{T_{0}}|\widehat{\mathscr{G}}(t, s, y)-\mathscr{G}(t, s, y)| \mathrm{d} s \\
& +\frac{\left|1-q_{*}\right|}{q_{*}} \int_{t}^{T} \mathbf{E}_{T_{0}}\left|\widehat{h}\left(s, \widehat{\eta}_{s}^{t, y}\right)-h\left(s, \eta_{s}^{t, y}\right)\right| e^{Q_{*}(s-t)} \mathrm{d} s .
\end{aligned}
$$

Учитывая, что $q_{*}=1 /(1-\gamma)>1$, и используя неравенства (10.9) и (10.10), находим

$$
\begin{aligned}
|\bar{h}(t, y)| \leqslant & (1+\widetilde{T}) \mathbf{E}_{T_{0}}|\widehat{\mathscr{G}}(t, T, y)-\mathscr{G}(t, T, y)| \\
& +\gamma \int_{t}^{T} \mathbf{E}_{T_{0}}\left|h\left(s, \widehat{\eta}_{s}^{t, y}\right)-h\left(s, \eta_{s}^{t, y}\right)\right| e^{Q_{*}(s-t)} \mathrm{d} s \\
& +\gamma \int_{t}^{T} \mathbf{E}_{T_{0}}\left|\widehat{h}\left(s, \widehat{\eta}_{s}^{t, y}\right)-h\left(s, \widehat{\eta}_{s}^{t, y}\right)\right| e^{Q_{*}(s-t)} \mathrm{d} s .
\end{aligned}
$$

Оценка сверху (10.5) влечет за собой неравенство

$$
\left|h\left(s, \widehat{\eta}_{s}^{t, y}\right)-h\left(s, \eta_{s}^{t, y}\right)\right| \leqslant h_{1}^{*}\left|\widehat{\eta}_{s}^{t, y}-\eta_{s}^{t, y}\right| .
$$

В силу определений метрики $\widetilde{\varrho}_{*}($ см. (10.12)) и параметра $\varkappa($ см. (4.2)) мы получаем

$$
\begin{aligned}
\widetilde{\varrho}_{*}(\widehat{h}, h) \leqslant & \frac{(1+\widetilde{T}) \widetilde{T} Q_{1}^{*}}{\left|\alpha_{1}\right|} \sup _{(t, y) \in \mathscr{K}}\left(\frac{\tilde{m}(y)}{\iota_{0}+|y|} e^{\left(Q_{*}-\varkappa\right)(T-t)}\right)|\widehat{\alpha}-\alpha| \\
& +\gamma \sup _{(t, y) \in \mathscr{K}} \int_{t}^{T} h_{1}^{*} \mathbf{E}_{T_{0}}\left|\widehat{\eta}_{s}^{t, y}-\eta_{s}^{t, y}\right| e^{\left(Q_{*}-\varkappa\right)(T-t)} \mathrm{d} s \\
& +\gamma \sup _{(t, y) \in \mathscr{K}} \int_{t}^{T} \mathbf{E}_{T_{0}} \frac{\left|\widehat{h}\left(s, \widehat{\eta}_{s}^{t, y}\right)-h\left(s, \widehat{\eta}_{s}^{t, y}\right)\right| e^{-\varkappa(T-s)}}{\iota_{0}+\left|\widehat{\eta}_{s}^{t, y}\right|} \\
& \times \frac{\iota_{0}+\left|\widehat{\eta}_{s}^{t, y}\right|}{\iota_{0}+|y|} e^{\left(Q_{*}-\varkappa\right)(s-t)} \mathrm{d} s .
\end{aligned}
$$

Тогда

$$
\begin{aligned}
\widetilde{\varrho}_{*}(\widehat{h}, h) & \leqslant \Upsilon^{*}|\widehat{\alpha}-\alpha|+\gamma \widetilde{\varrho}_{*}(\widehat{h}, h) \sup _{(t, y) \in \mathscr{K}} \int_{t}^{T} \frac{\iota_{0}+\mathbf{E}_{T_{0}}\left|\widehat{\eta}_{s}^{t, y}\right|}{\iota_{0}+|y|} e^{\left(Q_{*}-\varkappa\right)(s-t)} \mathrm{d} s \\
& \leqslant \Upsilon^{*}|\widehat{\alpha}-\alpha|+\gamma \widetilde{\varrho}_{*}(\widehat{h}, h) \sup _{(t, y) \in \mathscr{K}}\left(\frac{\iota_{0}+\widetilde{m}(y)}{\iota_{0}+|y|} \int_{t}^{T} e^{\left(Q_{*}-\varkappa\right)(s-t)} \mathrm{d} s\right) \\
& \leqslant \Upsilon^{*}|\widehat{\alpha}-\alpha|+\frac{2 \gamma}{\varkappa-Q_{*}} \widetilde{\varrho}_{*}(\widehat{h}, h),
\end{aligned}
$$


где $\Upsilon^{*}=\left(2 Q_{1}^{*} \widetilde{T}+\gamma h_{1}^{*}\right) \widetilde{T} /\left|\alpha_{1}\right|$. Следовательно,

$$
\widetilde{\varrho}_{*}(\widehat{h}, h) \leqslant \frac{\varkappa-Q_{*}}{\varkappa-Q_{*}-2 \gamma} \Upsilon^{*}|\bar{\alpha}| .
$$

Учитывая, что $\varkappa=Q_{*}+\zeta_{0}+2 \gamma+1$, получаем (10.13). Предложение 10.1 доказано.

Рассмотрим случай, когда оба параметра $\mu$ и $\alpha$ неизвестны. Следующая лемма дает аналог неравенства (10.9).

Лемма 10.7. Если параметры $\mu$, а неизвестны, то при любом $0<T_{0}<T$ имеет место оченка

$\mathbf{E}_{T_{0}}|\widehat{\mathscr{G}}(t, s, y)-\mathscr{G}(t, s, y)| \leqslant \gamma \frac{\mu_{2}+r+1}{(1-\gamma) \sigma_{1}^{2}} \widetilde{T} e^{Q_{*}(T-t)} \varpi+\widetilde{T} Q_{1}^{*} e^{Q_{*}(T-t)} \frac{\widetilde{m}(y)}{\left|\alpha_{1}\right|}|\bar{\alpha}|$,

где $\varpi=|\widehat{\mu}-\mu|+|\widehat{\mu}-\mu|^{2}$.

Д о к а з а т е л ь с т в о. Сначала напомним, что функция $Q$ определена в (3.4). Мы можем получить следующую верхнюю границу:

$$
\widehat{Q}(z)-Q(z)=\frac{\gamma\left(\widehat{\theta}^{2}(z)-\theta^{2}(z)\right)}{2(1-\gamma)} \leqslant \frac{\gamma\left(\mu_{2}+r+1\right)}{(1-\gamma) \sigma_{1}^{2}} \varpi
$$

Следовательно,

$$
\begin{aligned}
|\widehat{\mathscr{G}}(t, s, y)-\mathscr{G}(t, s, y)| \leqslant & \left|\exp \left\{\int_{t}^{s} \widehat{Q}\left(\widehat{\eta}_{u}^{t, y}\right) \mathrm{d} u\right\}-\exp \left\{\int_{t}^{s} Q\left(\widehat{\eta}_{u}^{t, y}\right) \mathrm{d} u\right\}\right| \\
& +e^{Q_{*}(T-t)}\left|\int_{t}^{s} Q\left(\widehat{\eta}_{u}^{t, y}\right) \mathrm{d} u-\int_{t}^{s} Q\left(\eta_{u}^{t, y}\right) \mathrm{d} u\right| \\
\leqslant & \widetilde{T} e^{Q_{*}(T-t)} \frac{\gamma\left(\mu_{2}+r+1\right)}{(1-\gamma) \sigma_{1}^{2}} \varpi \\
& +Q_{1}^{*} e^{Q_{*}(T-t)} \int_{t}^{T}\left|\widehat{\eta}_{u}^{t, y}-\eta_{u}^{t, y}\right| \mathrm{d} u .
\end{aligned}
$$

С помощью (10.8) мы получаем (10.14). Лемма 10.7 доказана.

Следующее предложение - это аналог предложения 10.1. Разница в том, что в предложении ниже оба параметра $\mu$ и $\alpha$ неизвестны.

Предложение 10.2. Для неизвестных параметров $\mu, \alpha$ и любых $0<T_{0}<T$ имеет место оченка

$$
\widetilde{\varrho}_{*}(\widehat{h}, h) \leqslant \mathbf{h}_{1}|\bar{\alpha}|+\mathbf{h}_{2} \varpi,
$$

где метрика $\widetilde{\varrho}_{*}$ определена в (10.12), а константь $\mathbf{h}_{1}$ и $\mathbf{h}_{2}$ определень в (8.9) и (8.20) соответственно.

Д о к а з а т е л с т в о. Мы рассуждаем так же, как при доказательстве предложения 10.1, и используем оценку для $\mathbf{E}_{T_{0}} \mid \widehat{\mathscr{G}}(t, T, y)$ $\mathscr{G}(t, T, y) \mid$ из леммы 10.7 . 


\section{СПИСОК ЛИТЕРАТУРЫ}

1. Berdjane B., Pergamenshchikov S. Optimal consumption and investment for markets with random coefficients. - Finance Stoch., 2013, v. 17, № 2, p. 419-446.

2. Castañeda-Leyva N., Hernández-Hernández D. Optimal consumption-investment problems in incomplete markets with stochastic coefficients. - SIAM J. Control Optim., 2005, v. 44, № 4, p. 1322-1344.

3. Delarue $F$. On the existence and uniqueness of solutions to FBSDEs in a nondegenerate case. - Stochastic Process. Appl., 2002, v. 99, № 2, p. 209-286.

4. Delong L., Klüppelberg C. Optimal investment and consumption in a Black-Scholes market with Lévy-driven stochastic coefficients. - Ann. Appl. Probab., 2008, v. 18, № 3, p. 879-908.

5. Fleming $W$., Hernández-Hernández D. An optimal consumption model with stochastic volatility. - Finance Stoch., 2003, v. 7, № 2, p. 245-262.

6. Fleming $W$., Rishel $R$. Deterministic and Stochastic Optimal Control. Berlin: Springer-Verlag, 1975, 222 p. (Appl. Math., v. 1.)

7. Fouque J.P., Papanicolaou G., Sircar R. Derivatives in Financial Markets with Stochastic Volatility. Cambridge: Cambridge Univ. Press, 2000, 201 p.

8. Freidlin M.I. Functional Integration and Partial Differential Equations. Princeton: Princeton Univ. Press, 1985, 545 p. (Ann. Math. Stud., v. 109.)

9. Hernández-Hernández D., Schied A. Robust utility maximization in a stochastic factor model. — Statist. Decisions, 2006, v. 24, № 1, p. 109-125.

10. Jackwerth J., Rubinstein M. Recovering probability distributions from option prices. - J. Finance, 1996, v. 51, № 5, p. 1611-1631.

11. Kabanov Yu. M., Pergamenshchikov S. M. Two-Scale Stochastic Systems. Asymptotic Analysis and Control. Berlin: Springer-Verlag, 2003, 266 p. (Appl. Math., v. 49.)

12. Karatzas I., Shreve S.E. Brownian Motion and Stochastic Calculus. New York: Springer, 1991, $470 \mathrm{p}$.

13. Karatzas I., Shreve S. E. Methods of Mathematical Finance. Berlin: Springer, 1998, $407 \mathrm{p}$.

14. Konev V., Pergamenshchikov $S$. On truncated sequential estimation of the parameters of diffusion processes. - Methods of Economical Analysis. Ed. by S. Aivazian. Moscow: Central Economical and Mathematical Institute of Russian Academy of Science, 1992, p. 3-31.

15. Korn R. Optimal Portfolios. Singapore: World Scientific, 1997, 338 p.

16. Kraft $H$., Steffensen $M$. Portfolio problems stopping at first hitting time with application to default risk. - Math. Methods Oper. Res., 2006, v. 63, № 1, p. 123-150.

17. Ладыженская O.А., Солонников В. А., Уральцева Н. Н. Линейные и квазилинейные уравнения параболического типа. М.: Наука, 1967, 736 с.

18. Liptser R. S., Shiryaev A. N. Statistics of Random Process. I: General Theory. Berlin: Springer-Verlag, 2001, $427 \mathrm{p}$.

19. Liptser R.S., Shiryaev A. N. Statistics of Random Process. II: Applications. Berlin: Springer-Verlag, 2001, $402 \mathrm{p}$.

20. Bardi M., Cesaroni A., Manca L. Convergence by viscosity methods in multiscale financial models with stochastic volatility. - SIAM J. Financial Math., 2010, v. 1, p. 230-265.

21. Ma J., Protter P., Yong J. Solving forward-backward stochastic differential equations explicitly — a four step scheme. - Probab. Theory Related Fields, 1994, v. 98, № 3, p. 339-359.

22. Merton $R$. Optimal consumption and portfolio rules in a continuous time model. J. Econom. Theory, 1971, v. 3, № 4, p. 373-413.

23. Новиков A. A. Последовательное оценивание параметров диффузионных процессов. (Резюме доклада, сделанного на заседании семинара по теории вероятностей и математической статистике в Математическом институте Академии наук CССР.) - Теория вероятн. и ее примен., 1971, т. 16, в. 2, с. 394-396.

24. Pazy A. Demigroups in Linear Operators and Applications to Partial Differential Equations. New York: Springer, 1983, 279 p. (Appl. Math. Sci., v. 44.) 
25. Pham H. Smooth solutions to optimal investment models with stochastic volatilities and portofolio constraints. - Appl. Math. Optim., 2002, v. 46, № 1, p. 55-78.

26. Presman E.L., Sethi S.P. Risk-aversion behavior in consumption/investment problems. - Math. Finance, 1991, v. 1, № 1, p. 100-124.

27. Rogers L. C. G. Optimal Investment. Berlin: Springer-Verlag, 2013, 156 p. (Springer Briefs Quant. Finance.)

28. Rubinstein $M$. Nonparametric tests of alternative option pricing models using all reported trades and quotes on the 30 most active CBOE option classes from August 23, 1976 through August 31, 1976. - J. Finance, 1985, v. 40, № 2, p. 455-480.

29. Sethi S.P., Taksar M.I., Presman E.L. Explicit solution of a general consumption/portfolio problem with subsistence consumption and bankruptcy. J. Econom. Dynam. Control, 1992, v. 16, № 3-4, p. 747-768.

30. Sircar R., Zariphopoulou T. Bounds and asymptotic approximations for utility prices when volatility is random. - SIAM J. Control Optim., 2005, v. 43, № 4, p. 1328-1353.

31. Zariphopoulou T. A solution approach to valuation with unhedgeable risks. - Finance Stoch., 2001, v. 5, № 1, p. 61-82.

Поступила в редакцию

2.III. 2015 